\title{
THE SMALLEST POSITIVE EIGENVALUE OF FIBERED HYPERBOLIC 3-MANIFOLDS
}

\author{
HYUNGRYUL BAIK, ILYA GEKHTMAN AND URSULA HAMENSTÄDT
}

\begin{abstract}
We study the smallest positive eigenvalue $\lambda_{1}(M)$ of the Laplace-Beltrami operator on a closed hyperbolic 3-manifold $M$ which fibers over the circle, with fiber a closed surface of genus $g \geq 2$. We show the existence of a constant $C>0$ only depending on $g$ so that $\lambda_{1}(M) \in\left[C^{-1} / \operatorname{vol}(M)^{2}, C \log \operatorname{vol}(M) / \operatorname{vol}(M)^{2^{2 g-2} /\left(2^{2 g-2}-1\right)}\right]$ and that this estimate is essentially sharp. We show that if $M$ is typical or random, then we have $\lambda_{1}(M) \in\left[C^{-1} / \operatorname{vol}(M)^{2}, C / \operatorname{vol}(M)^{2}\right]$. This rests on a result of independent interest about reccurence properties of axes of random pseudo-Anosov elements.
\end{abstract}

\section{Contents}

1. Introduction

2. The thick part of a mapping torus

3. Arrays of circles

4. The smallest eigenvalue of mapping tori 26

5. Typical mapping tori 29

6. Random mapping tori $\quad 31$

References

\section{INTRODUCTION}

The smallest positive eigenvalue $\lambda_{1}(M)$ of the Laplace-Beltrami operator on a closed Riemannian manifold $M$ equals the infimum of the Rayleigh quotients

$$
\lambda_{1}(M)=\inf _{f \in C_{m}^{\infty}(M)} \frac{\int_{M}\|\nabla f\|^{2} d M}{\int_{M} f^{2} d M},
$$

where $C_{m}^{\infty}(M)$ denotes the vector space of smooth functions $f$ on $M$ with $\int_{M} f d M=0$.

For closed hyperbolic surfaces $S$ of fixed genus $g \geq 2$ and hence of fixed volume, this eigenvalue can be arbitrarily close to zero if there is a separating short geodesic on $S$. In fact, $\lambda_{2 g-3}(S)$ can be arbitrarily small (Theorem

Date: August 26, 2016.

AMS subject classification: 58C40, 30F60, 20P05

Research of all three authors supported by ERC grant 10160104. 
8.1.3 of [6]). But for a closed hyperbolic 3-manifold $M$, Schoen [32] established the existence of a universal and explicit constant $b_{1}>0$ such that

$$
\lambda_{1}(M) \geq \frac{b_{1}}{\operatorname{vol}(M)^{2}} .
$$

The same lower bound holds true for hyperbolic 3-manifolds of finite volume [8].

On the other hand, Buser [5] showed that the so-called Cheeger constant $h(M)$ of $M$ can be used to give an upper estimate for $\lambda_{1}(M)$ by

$$
\lambda_{1}(M) \leq b_{2}\left(h(M)+h^{2}(M)\right)
$$

where $b_{2}>0$ is a universal constant (which in a more general setting depends on the dimension and a lower bound on the Ricci curvature).

Lackenby [17] related the Cheeger constant $h(M)$ to the Heegaard Euler characteristic $\chi_{H}(M)$ of $M$. He showed that

$$
h(M) \leq \frac{4 \pi\left|\chi_{H}(M)\right|}{\operatorname{vol}(M)} .
$$

If we denote by genus $(M)$ the more familiar Heegaard genus of $M$, then we have $\chi_{H}(M)=2-2 \operatorname{genus}(M)$.

Since there is a positive lower bound for the volume of a hyperbolic 3manifold, these results can be summarized as follows. For every $g>0$ there exists a constant $b_{3}(g)>0$ with the following property. Let $M$ be a closed hyperbolic 3-manifold of Heegaard genus at most $g$; then

$$
\frac{b_{1}}{\operatorname{vol}(M)^{2}} \leq \lambda_{1}(M) \leq \frac{b_{3}(g)}{\operatorname{vol}(M)} .
$$

For manifolds $M$ with a given lower bound of the injectivity radius, there is more precise information. Namely, White [34] proved that there exists a number $b_{4}=b_{4}(g, \varepsilon)>0$ such that

$$
\lambda_{1}(M) \leq \frac{b_{4}(g, \varepsilon)}{\operatorname{vol}(M)^{2}}
$$

for all closed hyperbolic 3-manifolds of Heegaard genus at most $g$ and injectivity radius at least $\varepsilon$. The existence of expander families yield that the dependence of $b_{4}(g, \varepsilon)$ on $g$ is necessary. We refer to [13] for a more complete discussion.

In this work we are interested in $\lambda_{1}(M)$ for a closed hyperbolic threemanifold $M$ which fibers over the circle, with fiber a closed surface $S$ of genus $g \geq 2$. Such a manifold can be described as a mapping torus of a pseudo-Anosov diffeomorphism of $S$, in particular, there are infinitely many such mapping tori. The Heegaard genus of a mapping torus of genus $g$ is not bigger than $2 g+1$ (see [17] for references).

Our first goal is to give an essentially sharp upper bound for $\lambda_{1}(M)$ for hyperbolic mapping tori $M$ of fibre genus $g$. We prove. 
Theorem 1. For every $g \geq 2$ there exists a constant $C_{1}=C_{1}(g)>0$ with the following property.

(1) Let $M$ be a hyperbolic mapping torus of genus $g$; then

$$
\lambda_{1}(M) \leq \frac{C_{1} \log \operatorname{vol}(M)}{\operatorname{vol}(M)^{2^{2 g-2} /\left(2^{2 g-2}-1\right)}} .
$$

(2) There exists a sequence $M_{i}$ of hyperbolic mapping tori of genus $g$ with $\operatorname{vol}\left(M_{i}\right) \rightarrow \infty$ such that

$$
\lambda_{1}\left(M_{i}\right) \geq \frac{C_{1}^{-1}}{\operatorname{vol}\left(M_{i}\right)^{2^{2 g-2} /\left(2^{2 g-2}-1\right)}} .
$$

We believe that Theorem 1 easily generalizes to hyperbolic mapping tori of non-exeptional surfaces of finite type with punctures, but we did not check the details. By the work of White [34, the injectivity radius of the examples in the second part of the above theorem tends to zero with $i$.

The estimates in part (1) and (2) of the theorem differ by a factor $\log \operatorname{vol}(M)$. This deviation arises as follows. Any closed hyperbolic 3manifold $M$ admits a thick-thin decomposition $M=M_{\text {thick }} \cup M_{\text {thin }}$ where for some small but fixed number $\varepsilon>0, M_{\text {thin }}$ consists of all points of injectivity radius smaller than $\varepsilon$, and $M_{\text {thick }}=M-M_{\text {thin }}$.

We estimate effectively the smallest eigenvalue $\lambda_{1}\left(M_{\text {thick }}\right)$ of $M_{\text {thick }}$ with Neumann boundary conditions as a function of the volume. We then use a result of [13]: There exists a universal constant $b>0$ such that

$$
b^{-1} \lambda_{1}\left(M_{\text {thick }}\right) \leq \lambda_{1}(M) \leq b \log \operatorname{vol}\left(M_{\text {thin }}\right) \lambda_{1}\left(M_{\text {thick }}\right)
$$

for every closed hyperbolic 3-manifold $M$. The factor $\log \operatorname{vol}(M)$ in the statement of the first part of Theorem 1 arises from the ratio $\lambda_{1}(M) / \lambda_{1}\left(M_{\text {thick }}\right)$.

Although there is a sequence $M_{i}$ of hyperbolic mapping tori of genus $g$ with $\operatorname{vol}\left(M_{i}\right) \rightarrow \infty$ and $\lambda_{1}\left(M_{i}\right) \geq b^{\prime} \log \operatorname{vol}\left(M_{i}\right) \lambda_{1}\left(\left(M_{i}\right)_{\text {thick }}\right)$ where $b^{\prime}>0$ is another universal constant 13, we do not know whether such a sequence exists which moreover satisfies $\lambda_{1}\left(\left(M_{i}\right)_{\text {thick }}\right) \geq b^{\prime \prime} / \operatorname{vol}\left(M_{i}\right)^{2^{2 g-2} /\left(2^{2 g-2}-1\right)}$ for a universal constant $b^{\prime \prime}>0$.

Most mapping tori $M$, however, have $\lambda_{1}(M)$ proportional to $1 / \operatorname{vol}(M)^{2}$. We make this precise in the following result. Let from now on $S$ be a closed surface of genus $g \geq 2$.

A hyperbolic mapping torus is determined up to isometry by the conjugacy class in the mapping class group $\operatorname{Mod}(S)$ of a defining pseudo-Anosov element. Conjugacy classes in $\operatorname{Mod}(S)$ can be listed according to their translation length. Call a property $\mathcal{P}$ for hyperbolic mapping tori typical if the proportion of the number of conjugacy classes of pseudo-Anosov elements of translation length at most $L$ which give rise to a 3 -manifold with this property tends to one as $L \rightarrow \infty$. We refer to Section 5 for a more detailed discussion. We then say that a typical mapping torus has property $\mathcal{P}$.

Similarly, we say that a random mapping torus has property $\mathcal{P}$ if a statistical point for a random walk on $\operatorname{Mod}(S)$ induced by a probability measure 
on $\operatorname{Mod}(S)$ whose finite support generates all of $\operatorname{Mod}(S)$ defines a mapping torus with this property. Answering a question of Rivin [31] we show

Theorem 2. For every $g \geq 2$ there is a constant $C_{2}=C_{2}(g)>0$ so that the following holds true. Let $M$ be a typical or random mapping torus of genus $g$; then

$$
\lambda_{1}(M) \leq \frac{C_{2}}{\operatorname{vol}(M)^{2}} .
$$

The proof of Theorem 2 for random mapping tori uses the groundbreaking work of Minsky [28] and Brock, Canary and Minsky [4] and recurrence properties of random walks on the mapping class group $\operatorname{Mod}(S)$ acting on Teichmüller space $\mathcal{T}(S)$ which are of independent interest. In the formulation of our main result on random walks, we use the following notation. For a pseudo-Anosov mapping class $\phi$, we denote by $\ell(\phi)$ the translation length of $\phi$ for its action on $\mathcal{T}(S)$ (which coincides with the translation length on its axis $\left.\gamma_{\phi}\right)$. For a number $\zeta>0$ and a subset $U$ of $\mathcal{T}(S)$ let moreover $N_{\zeta}(U)$ be the $\zeta$-neighborhood of $U$ with respect to the Teichmüller distance. We prove.

Theorem 3. There exists a number $\zeta=\zeta(g)$ with the following property. Let $\mu$ be a nonelementary finitely supported probability measure on the mapping class group. Let $U \subset \mathcal{T}(S)$ be an $\operatorname{Mod}(S)$ invariant open subset which contains the axis of at least one pseudo-Anosov element. Then for each $p>0$, there exists $c=c(U, p)>0$ such that

$$
\begin{aligned}
& \mu^{* n}\{\phi \in \operatorname{Mod}(S) \mid \phi \text { is } p \text { - } A \text { and } \\
& \left.l(\phi)^{-1}\left|\left\{t \in[0, l(\phi)): \gamma_{\phi}(t-p, t+p) \subset N_{\zeta} U\right\}\right|>c\right\} \rightarrow 1 \quad(n \rightarrow \infty)
\end{aligned}
$$

Different but related recurrence properties for axes of random pseudoAnosov elements have been obtained independently at the same time by Gadre and Maher in [9].

The proof of this result rests on a technical tool (Proposition 6.11) which states that for typical trajectories of the random walk, axes of pseudoAnosov elements in Teichmüller space fellow travel rays from a basepoint. We refer to [7] to closely related earlier work.

The organization of the paper is as follows. In Section 2 we use the work [28, 4] of Minsky and Brock, Canary and Minsky to determine a collection of graphs with the property that the thick part of every hyperbolic mapping torus of genus $g$ is uniformly quasi-isometric to a graph in the collection.

Section 3 is devoted to estimating the first eigenvalues of these graphs as a function of their volume. By the main result of [22], the smallest positive eigenvalue of the thick part of a mapping torus with Neumann boundary conditions can be estimated in the same way. Theorem 1 follows from this fact and [13] as explained in Section 4. The proof of Theorem 2 for typical mapping tori is contained in Section 5. Section 6 is devoted to studying geometric properties of random walks on the mapping class group, with the proof of Theorem 3 as the main goal. 
Acknowledgement: We are all very grateful to Juan Souto for helpful discussions. A version of Theorem 1 is due independently to Anna Lenzhen and Juan Souto [18. We are also grateful to Samuel Taylor for pointing out a gap in the proof of Proposition 5.1 of a previous version of the paper.

\section{THE THICK PART OF A MAPPING TORUS}

A closed hyperbolic 3-manifold $M$ admits a thick-thin decomposition

$$
M=M_{\text {thin }} \cup M_{\text {thick}} .
$$

The thin part $M_{\text {thin }}$ is the set of all points $x$ with injectivity $\operatorname{radius} \operatorname{inj}(x) \leq \varepsilon$ where $\varepsilon>0$ is sufficiently small but fixed, and $M_{\text {thick }}=\{x \mid \operatorname{inj}(x) \geq \varepsilon\}$. For an appropriate choice of $\varepsilon, M_{\text {thick }}$ is not empty and connected, and $M_{\text {thin }}$ is a union of (at most) finitely many Margulis tubes. Such a Margulis tube is diffeomorphic to a solid torus, and it is a tubular neighborhood of a closed geodesic of length at most $2 \varepsilon$. This geodesic is called the core curve of the tube.

The goal of this section is to establish an understanding of the geometric shape of the thick part of a hyperbolic mapping torus $M$ of genus $g$. To such a mapping torus $M$, Minsky 28] associates a combinatorial model which is quasi-isometric to $M$. We use this model to construct a graph which is $L$ quasi-isometric to $M_{\text {thick }}$ for a number $L>1$ only depending on $g$. These graphs will be used in Section 3 and Section 4 for the proof of Theorem 1.

Furthermore, under some additional assumption on $M$, we construct geometrically controlled submanifolds in $M_{\text {thick }}$ with boundary. These submanifolds will be used to estimate the smallest positive eigenvalue of random mapping tori.

The results in this section heavily depend on the results in [28, 4] of Minsky and Brock, Canary and Minsky. The reader who is not familiar with the ideas developed in [28, 4] will however have no difficulty to understand the statement of Proposition 2.2 which is all what is needed for the proof of Theorem 1.

We begin with introducing the class of graphs we are interested in. By a graph we always mean a finite connected graph $G$. We equip $G$ with a metric so that each edge of $G$ has length one. An arc in a graph $G$ is a connected subgraph of $G$ which is homeomorphic to an interval. The length of the arc is the number of its edges. The length of an arc is at least one. If $a$ is an arc of length $k$ then $a$ contains $k-1$ vertices of valence two and two endpoints which are vertices of valence one. A circle is a finite connected graph $L$ with all vertices of valence two. Then $L$ is homeomorphic to $S^{1}$. Its length equals the number of its edges. We always assume that the length of a circle is at least two. We say that a subgraph $G_{1}$ of a graph $G$ is attached to a subgraph $G_{2}$ of $G$ at a vertex $v$ if $G_{1} \cap G_{2}=\{v\}$.

Definition 2.1. For $h \geq 1$, an array of circles of depth at most $h$ is a finite connected graph $G$ of the following form. $G$ contains a subgraph $L$ which is 
a circle called a base circle. If $h=1$ then $G=L$. Otherwise $G$ is obtained from $L$ by attaching to each vertex of $L$ an array of circles of depth at most $h-1$. The depth of an array of circles $G$ is defined to be the smallest number $h$ so that $G$ is of depth at most $h$.

Note that a bouquet of two circles is an array of circles of depth two, but both circles may be used as the base circle, so the base circle may not be uniquely determined and hence a given graph may admit more than one description as an array of circles. In the sequel, whenever we speak of an array of circles, we assume that one choice of such a description has been made.

Closely related to arrays of circles is a more general class of graphs which we call generalized arrays of circles. These are finite connected graphs whose construction is by induction on a notion of depth $h$ as follows.

If $h=1$ then $G$ is simply a circle. In the case $h \geq 2$ we begin as before with a circle $L$. Given a vertex $v$ of $L$, we allow to either attach to $v$ a generalized array of circles of depth at most $h-1$, or we allow to replace $v$ by a graph consisting of $2 \leq s \leq h$ arcs $a_{1}, \ldots, a_{s}$ of possibly distinct length with disjoint interior and with the same pair of distinct endpoints. If $v_{1} \neq v_{2}$ are these endpoints, then the graph obtained by identifying $v_{1}$ and $v_{2}$ is just the base circle $L$ with $s$ circles attached at $v$. We call the arcs $a_{1}, \ldots, a_{s}$ vertex arcs, and we call the vertex $v$ of $L$ which was replaced by $a_{1}, \ldots, a_{s}$ in this way a blown-up vertex.

We require furthermore that for each blown-up vertex with corresponding set $a_{1}, \ldots, a_{s}$ of vertex arcs, there is a decomposition $h=\sum_{i=1}^{s} m_{i}$ where $m_{i} \geq 1$. By induction, we allow to attach to each interior vertex of an arc $a_{i}$ a generalized array of circles of depth at most $m_{i}-1$. This also includes the possibility that this interior vertex is blown up to $u \leq m_{i}$ arcs with the same endpoints as described above.

As an example, if the depth of the generalized array of circles $G$ equals two then $G$ is obtained from the base circle $L$ by either attaching to a vertex of $L$ a (possibly trivial) circle or by replacing the vertex by two arcs of possibly different length, and these possibilities are mutually exclusive.

Proposition 2.2. There is a number $L=L(g, \varepsilon)>0$ with the following property. Let $M$ be a hyperbolic mapping torus of genus $g$. Then $M_{\text {thick }}$ is $L$-quasi-isometric to a generalized array of circles of depth at most $2 g-2$.

Proof. Let $\hat{M}$ be the infinite cyclic cover of $M$ defined by the fibration $M \rightarrow S^{1}$. Its deck group is generated by a pseudo-Anosov diffeomorphism $\phi: S \rightarrow S$ whose mapping torus is $M$. Fix a homotopy equivalence $S \rightarrow \hat{M}$.

Part of the main result of [28, 4] can be summarized as follows.

There exists a model manifold $N$ for $\hat{M}$ which is homeomorphic to $S \times \mathbb{R}$ and is composed of combinatorial pieces called blocks. This model manifold admits an infinite cyclic group of homeomorphisms compatible with the block decomposition which is generated by a homeomorphism $\psi: N \rightarrow N$. The quotient $N /\langle\psi\rangle$ is homeomorphic to $M$. 
Within $N$ there is a $\psi$-invariant subset $\mathcal{U}$ which consists of open solid tori of the form $U=A \times J$, where $A$ is an annulus in $S$ and $J$ is an interval in $\mathbb{R}$. The manifold $N$ is equipped with a $\psi$-invariant piecewise smooth Riemannian metric. The induced metric on the boundary $\partial U$ of each $U \in \mathcal{U}$ is flat. The geometry of the flat torus $\partial U$ is described by a coefficient $\omega_{N}(U) \in \mathbf{H}^{2}$, where $\mathbf{H}^{2}$ is thought of as the Teichmüller space of the two-torus (i.e., the space of marked flat metrics on the two-torus). For $k \geq 1$ let $\mathcal{U}[k]$ denote the union of the components of $\mathcal{U}$ with $\left|\omega_{N}\right| \geq k$ and let $N[k]=N-\mathcal{U}[k]$.

The following statement is a combination of the Lipschitz Model Theorem and the Short Curve Theorem as stated in the introduction of [28], and the Bilipschitz Model Theorem from Section 8 of [4].

There exist numbers $K, k>0$ only depending on the genus of $S$ but not on the mapping torus $M$, and there is a $\psi-\phi$-equivariant $K$-Lipschitz map $F: N \rightarrow \hat{M}$ with the following properties.

(1) $F$ induces a marked isomorphism $\pi_{1}(N)=\pi_{1}(S) \rightarrow \pi_{1}(\hat{M})$, is proper and has degree one.

(2) $F$ is $K$-bilipschitz on $N[k]$ with respect to the induced path metric.

(3) $F$ maps each component of $\mathcal{U}[k]$ to a Margulis tube, and each Margulis tube with sufficiently short core curve is contained in the image of a component of $\mathcal{U}[k]$.

Thus all we need to show is that for the number $k$ in the above statement, $N[k]$ is $L$-quasi-isometric to a generalized array of circles for a universal number $L>0$. This statement in turn follows from the construction of the model manifold which we outline next (we refer to [28] for all details).

A clean marking of the surface $S$ consists of a pants decomposition $P$ of $S$, the so-called base of the marking, and a set of so-called spanning curves. For each pants curve $c \in P$, there exists a unique spanning curve. This spanning curve is contained in $S-(P-c)$, and it intersects $c$ transversely in one or two points depending on whether the component of $S-(P-c)$ containing $c$ is a one-holed torus or a four-holed sphere.

A pants decomposition $P$ of $S$ is short in $M$ if there is a map $F: S \rightarrow M$ in the given homotopy class which maps each component of $P$ to a geodesic in $M$ of uniformly bounded length.

To build the model manifold, start with a pants decomposition $P$ which is short in $M$. Since each point in $M$ is uniformly near a pleated surface $f:(S, \sigma) \rightarrow \hat{M}$ (see Theorem 3.5 of [26] for this result of Thurston), short pants decompositions exist. Namely, such a pleated surface $f$ is a path isometry for a hyperbolic metric $\sigma$ on $S$. Furthermore, for every hyperbolic metric on $S$ there is a pants decomposition of uniformly bounded length.

A pants decomposition of $S$ can be viewed as a maximal simplex in the curve complex $\mathcal{C}(S)$ of $S$. By Theorem 6.1 and Theorem 7.1 of [28], we may assume that a short pants decomposition in $M$ is a simplex in $\mathcal{C}(S)$ which is uniformly near (for the distance in $\mathcal{C}(S)$ ) to a curve in any choice 
of a hierarchy constructed from the ending laminations of $\hat{M}$. These ending laminations are just the supports of the horizontal and vertical measured geodesic laminations on $S$ which determine the axis of the pseudo-Anosov mapping class $\phi$.

The vertical measured geodesic lamination $\lambda$ of the axis of $\phi$ determines an essentially unique clean marking $\mu$ of $S$ with base the given pants decomposition $P$. The spanning curves are determined by the subsurface projection of $\lambda$ into the collars of $P$. We refer to [25, 28, for details of this construction.

Let $\phi(\mu)$ be the image of $\mu$ under $\phi$. To $\mu$ and $\phi(\mu)$ we can associate a hierarchy $H$ and a resolution of $H$. This hierarchy consists of a collection of so-called tight geodesics in the curve complex of connected subsurfaces of $S$ different from three-holed spheres. The hierarchy is required to be four-complete. This means the following.

Define the complexity $\xi(Y)$ of a connected subsurface $Y$ of $S$ of genus $h \geq 0$ with $b \geq 0$ boundary components by $\xi(Y)=3 h+b$. Suppose that $Y \subset S$ is a complementary component of a vertex in a geodesic $h$ from the hierarchy $H$ whose domain is a surface $Y^{\prime} \supset Y$. If $\xi(Y) \geq 4$ then $Y$ is the domain of a geodesic in $H$.

Choose such a four-complete hierarchy $H$ associated to $\mu$ and $\phi(\mu)$ as well as a resolution of $H$. Each edge $e$ in a geodesic from the hierarchy $H$ whose domain $D(e)$ satisfies $\xi(D(e))=4$ (i.e. $D(e)$ either is a four-holed sphere or a one-holed torus) defines a block $B(e)$ for the component domain $D(e)$. The backward endpoint $e_{-}$of $e$ and the forward endpoint $e_{+}$can be identified with a simple closed curve in the component domain $D(e)$ of distance one in the curve graph of $D(e)$.

The block $B(e)$ for the edge $e$ is then defined as

$$
B(e)=(D(e) \times[-1,1])-\left(\operatorname{collar}\left(e_{-}\right) \times[-1,-1 / 2) \cup \operatorname{collar}\left(e_{+}\right) \times(1 / 2,1]\right) .
$$

The glueing boundary of the block $B(e)$ is defined to be

$$
\partial_{ \pm} B(e)=\left(D(e)-\operatorname{collar}\left(e^{ \pm}\right)\right) \times\{ \pm 1\} .
$$

This glueing boundary is a union of three-holed spheres.

There are only two combinatorial types of blocks [28]. Each block can be equipped with a standard Riemannian metric with totally geodesic boundary so that combinatorially equivalent blocks are isometric. The blocks are glued along the components of their glueing boundaries as prescribed by the resolution of the hierarchy $H$ and such that the metrics on the glueing boundaries of the blocks match up. Let $N[0]$ be these glued blocks (compare [28] for notation). Then $N[0]$ is a Riemannian manifold whose boundary is a union of two-dimensional tori.

Each boundary torus $\partial U$ contains a distinguished free homotopy class of simple closed geodesics, the predicted meridians. Such a predicted meridian is given as follows. There is a simple closed curve $v$ on $S$ so that for any simple arc $a$ on $S$ connecting the boundary components of a collar neighborhood of $v$, the predicted meridian equals $\partial(a \times[s, t])$ where the parameters 
$s<t$ can be read off from the hierarchy (we refer to p.80 of [28] for details). This predicted meridian and the simple closed curve $v$ of length $t=\varepsilon$ (here as before, $\varepsilon>0$ is a fixed Margulis constant) determine $\partial U$ as a marked flat torus. Following Section 3.2 of [28], these data also determine uniquely a meridian coefficient $\omega_{N}(\partial U) \in \mathbf{H}^{2}$. The length of the predicted meridian of the torus equals $\varepsilon\left|\omega_{N}(\partial U)\right|$, and the imaginary part $\Im \omega$ equals $1 / \varepsilon$ times the sum of the heights of the annuli that make up $\partial U$ (see p.80 of [28]).

Glue solid tori to those boundary tori $\partial U$ with coefficient $\left|\omega_{N}(U)\right| \leq k$. Up to isotopy, there is a unique way of such a glueing which maps the meridian of the solid torus to the predicted meridian of the boundary torus (compare again [28] for details). The resulting manifold $N[k]$ is the model for $M_{\text {thick }}$. Thus we have to verify that indeed, $N[k]$ is $L$-quasi-isometric to a generalized array of circles of depth at most $2 g-2$ for some $L>0$ only depending on $g$. Since the diameters of the tubes in $N[k]-N[0]$ are uniformly bounded, for this it suffices to show that $N[0]$ is uniformly quasiisometric to a generalized array of circles of depth at most $2 g-2$.

The pseudo-Anosov map $\phi$ maps the marked surface $(S, \mu)$ to $(S, \phi(\mu))$. By equivariance, there is a distinguished main tight geodesic $g_{H}$ in the hierarchy whose domain is the surface $S$ and which connects the marking $\mu$ to $\phi(\mu)$. This tight geodesic consists of a sequence of simplices $\left(v_{i}\right)$ in the curve complex $\mathcal{C}(S)$ of $S$ so that for any vertices $w_{i}$ of $v_{i}, w_{j}$ of $v_{j}$ we have $d_{\mathcal{C}_{1}(S)}\left(w_{i}, w_{j}\right)=|i-j|$ (here $d_{\mathcal{C}_{1}(S)}$ is the distance function of the one-skeleton of $\mathcal{C}(S))$. Moreover, for all $i, v_{i}$ represents the boundary of the subsurface of $S$ filled by $v_{i-1} \cup v_{i+1}$.

Glue the two ends of the tight geodesic $g_{H}$ using the map $\phi$ and view it as the base circle $L$ of a generalized array of circles. The length of $L$ equals the length of the tight geodesic $g_{H}$.

We use the resolution of the hierarchy to construct from $L$ a generalized array of circles as follows. A vertex $v_{i}$ of $g_{H}$ decomposes $S$ into complementary regions. Such a region $Y$ is a component domain of the hierarchy. If $Y$ is a component of $S-v_{i}$ different from a three-holed sphere then $v_{i-1} \mid Y$ and $v_{i+1} \mid Y$ are either markings of $Y$ or empty since $v_{i}$ is the boundary of the subsurface filled by $v_{i-1} \cup v_{i+1}$ (we ignore here the modification needed for the first and last simplex). In the case that $v_{i-1} \mid Y$ and $v_{i+1} \mid Y$ are both markings of $Y$, the hierarchy contains a tight geodesic with domain $Y$ connecting these markings.

As an example, if $Y$ is a connected subsurface of $S$ and if the subsurface projection [25] of $\mu \cup \phi(\mu)$ into $Y$ (which is never empty since $\mu$ and $\phi(\mu)$ are markings of $S$ ) has large diameter, then $Y$ arises as a component domain in the hierarchy. If $v_{i}$ is the simplex in the hierarchy with $Y$ as component domain, then the adjacent simplices $v_{i-1}$ and $v_{i+1}$ in the geodesic of the hierarchy containing $v_{i}$ fill $Y$ [25]. Thus there is a geodesic in the hierarchy with domain $Y$, and the length of this geodesic coincides with the diameter of this subsurface projection up to a universal additive constant (Lemma 5.9 of [28] summarizes this result from [25]). Even if $v_{i-1}$ and $v_{i+1}$ do not 
intersect the component $Y$ of $S-v_{i}$, if $Y$ is different from a three holed sphere then there is a geodesic in the hierarchy whose domain equals $Y$. As this will not be important for our purpose, we refer to [28] for a discussion of these technicalities.

For each $i$ let $u_{i}$ be the vertex of the circle $L$ corresponding to the simplex $v_{i}$. Let $Y_{1}, \ldots, Y_{s}$ be the complementary regions of $v_{i}$ in $S$. Let $a_{i}$ be the length of the tight geodesic in the hierarchy with domain $Y_{i}$. If $Y_{i}$ is a threeholed sphere then we define $a_{i}=1$. Blow up the vertex $u_{i}$ and replace it by $s$ $\operatorname{arcs}$ of length $a_{i}$. Moreover, associate to the arc $a_{i}$ the absolute value $-\chi\left(Y_{i}\right)$ of the Euler characteristic of $Y_{i}$. Note that $-\sum \chi\left(Y_{i}\right)=-\chi(S)=2 g-2$.

Each arc $a_{i}$ corresponds to a tight geodesic in a surface $Y_{i}$ of Euler characteristic $\chi\left(Y_{i}\right)$. Repeat the above construction with these arcs, successively blowing up vertices. Inductively, this defines a generalized array of circles of depth at most $2 g-2$.

To summarize, from the mapping torus we obtain (non-uniquely) a hierarchy $H$ and a resolution of $H$. The resolution is used to construct a generalized array of circles $G$ of depth at most $2 g-2$. There is natural map $\Psi: N[0] \rightarrow G$ which maps a block in $N[0]$ to an edge of $G$.

We are left with showing that this generalized array of circles indeed is uniformly quasi-isometric to $N[0]$. To this end simply recall that in the situation at hand, there are only two types of blocks [28. The first type of blocks is obtained from a component domain $D$ which is a one-holed torus. In the construction of the generalized array $G$, a geodesic $\eta$ in the hierarchy $H$ with a one-holed torus as component domain gives rise to an outmost arc, i.e. an arc of biggest depth. In the model manifold, it corresponds to a chain of blocks whose length equals the length of $\eta$.

In the natural order of blocks in the chain given by an orientation of the Teichmüller geodesic which defines the mapping torus, the top component of the glueing boundary of the last block in the chain consists of one threeholed sphere. This sphere is glued to the glueing boundary of a block $B$ which arises from a different geodesic of the hierarchy. The block $B$ is of the second type, obtained from a component domain which is a four-holed sphere. Then the second three-holed sphere in the glueing boundary of the block $B$ lying on the same "side" is glued to a block arising from a different geodesic in the hierarchy. This three-holed sphere may already be present at the initial point of the geodesic.

In the generalized array of circles, this corresponds precisely to glueing the endpoints of the arc representing the geodesic to the endpoints of another arc. The discussion of geodesics in $H$ whose component domains are fourholed spheres is completely analogous and will be omitted.

As a consequence, the map which associates to a block in $N[0]$ the edge of the generalized array of circles $G$ corresponding to it is essentially the map which associates to the decomposition of $N[0]$ into blocks the dual graph. The deviation from this precise picture comes from the addition of some additional edges in $G$, one for each "reassembling point", to meet the 
requirement of a generalized array of circles which we found most useful for our purpose. The proposition is proven.

Remark 2.3. The above construction also yields the following. Assume that for some $\delta>0, M$ is a mapping torus defined by a pseudo-Anosov mapping class whose axis is entirely contained in the $\delta$-thick part of Teichmüller space. Then $M$ is $L(\delta)$-quasi-isometric to a circle where $L(\delta)>0$ only depends on $\delta$.

More generally, let $M$ be a hyperbolic mapping torus of genus $g$ which is defined by a pseudo-Anosov mapping class $\phi$ of translation length $\ell$ in Teichmüller space. Let us assume that for some fixed number $c_{1} \in(0,1)$, the translation length for the action of $\phi$ on the curve graph of the surface of genus $g$ is at least $c_{1} \ell$. Then the length of the base circle of the array of circles constructed from $M$ in the proof of Proposition 2.2 is at least $c_{2} \operatorname{vol}\left(M_{\text {thick }}\right)=c_{3} \operatorname{vol}(M)$ where $c_{2}, c_{3}>0$ only depend on $c$ and $g$ (compare the discussion in [13] for a comparison between $\operatorname{vol}\left(M_{\text {thick }}\right)$ and $\left.\operatorname{vol}(M)\right)$.

Remark 2.4. From the model and the construction of a generalized array of circles $G$, we obtain some information of the size of the Margulis tubes in the mapping torus $M$. Namely, Margulis tubes with boundary of large volume correspond to blown-up vertices in the construction of $G$ with at least one long vertex arc.

However, large subsurface projections into the complement of a nonseparating simple closed curve on $S$ give rise to Margulis tubes which can not be detected in the generalized array of circles. Thus the thick-thin decomposition of $N$ can not be read off from the generalized array of circles.

As a consequence, a base circle of length $\sim \operatorname{vol}(M)$ does not imply that translation length for the action of the corresponding pseudo-Anosov element on the curve graph is proportional to its translation length on Teichmüller space.

For the proof of the second part of Theorem 1 we construct collections of mapping tori with fibre genus $g$ which are uniformly quasi-isometric to specific generalized arrays of circles.

We first introduce the arrays of circles we are interested in. Namely, let $G$ be an array of circles with base circle $L$ and depth $h$. Define the depth of a circle $C$ in $G$ as the minimal depth of an array of circles $G^{\prime} \subset G$ with base circle $L$ which contains $C$. Thus each circle in an array of circles of depth $h$ has depth at most $h$, and the base circle is the unique circle of depth one.

Call an array of circles $G$ step-homogeneous if all circles in $G$ of the same depth are non-degenerate and of the same length. A special example of a step-homogeneous array of circles is an array where for some $k \geq 2$, the circles of depth $\ell$ have length $k^{2^{\ell-1}}$. We call this array optimal. Note that an optimal array of circles is uniquely determined by the length $k$ of its base circle and by its depth. 
Proposition 2.5. For each $g>0$ there is a number $c_{1}(g)>0$ with the following properties. For $k \geq 2$ let $G$ be an optimal step-homogeneous array of circles of depth $2 g-2$, with base circle of length $k$. Then there is a mapping torus $M$ of genus $g$ so that $M_{\text {thick }}$ is $c_{1}(g)$-quasi-isometric to $G$.

Proof. Choose a decomposition of $S$ as a descending sequence of connected subsurfaces $S=S_{0} \supset S_{1} \supset \cdots \supset S_{2 g-3}$ with the following properties.

(1) $\chi\left(S_{i}\right)=2-2 g+i$.

(2) $S_{2 g-3}$ is a one-holed torus.

Such a chain can for example be constructed as follows. Choose a simple closed curve $\alpha_{1}$ which decomposes $S$ into a one-holed torus and a surface $S_{1}$ of genus $g-1$ with connected boundary. Choose a pair $\left(\alpha_{2}, \alpha_{2}^{\prime}\right)$ of simple closed curves which decompose $S_{1}$ into a three-holed sphere and a surface $S_{2}$ of genus $g-2$ with two boundary components. One proceeds inductively by decomposing $S_{i}$ into a three-holed sphere and a surface $S_{i+1}$ until $S_{i+1}$ becomes a one-holed torus. Since a one-holed torus has Euler characteristic -1 , the chain ends with the index $2 g-3$. Let $\mathcal{C}\left(S_{i}\right)$ be the curve complex of $S_{i}$

The union of the boundary circles of the surfaces $S_{i}$ is a pants decomposition $P$ of $S$. Let $\tau$ be a train track in standard form for $P$. We can choose $\tau$ in such a way that it restricts to a train track in standard form on each of the subsurfaces $S_{i}$ (here we have to be a bit careful what this means as $S_{i}$ has boundary). For terminologies regarding train tracks, we refer the readers to 29. In particular, one can find the definition of train track in standard form for a pants decomposition of $S$ and the proof of its existence in Sections 2.6 and 2.7 of [29].

For a subsurface $Y$ of $S$, recall that the mapping class $\operatorname{group} \operatorname{Mod}(Y)$ of $Y$ consists of isotopy classes of diffeomorphisms of $Y$ which fix the boundary of $Y$ pointwise. For each of the subsurfaces $S_{i}(0 \leq i \leq 2 g-3)$ choose once and for all a pseudo-Anosov mapping class $\phi_{i} \in \operatorname{Mod}\left(S_{i}\right)$ with the following properties.

(1) $\phi_{i}$ admits $\tau \mid S_{i}$ as a train track expansion.

(2) $d_{\mathcal{C}_{1}\left(S_{i}\right)}\left(c, \phi_{i}(c)\right) \geq 5$ for every simple closed curve $c$ on $S_{i}$.

Here as before, $d_{\mathcal{C}_{1}\left(S_{i}\right)}$ is the distance in the one-skeleton of the curve complex of the subsurface $S_{i}$.

The existence of $\phi_{i}$ satisfying (1) is a consequence of the fact that $\tau \mid S_{i}$ is maximal birecurrent which follows from the requirement that $\tau \mid S_{i}$ is in the standard form (as shown in [29]). (2) can be easily satisfied by first taking some $\phi_{i}$ satisfying (1) and replacing it by some positive power. Namely, by 24, for any pseudo-Anosov map $f$ on a non-exceptional surface $\Sigma$ and for any simple closed curve $\alpha$ on the surface, the $\operatorname{limit}_{n \rightarrow \infty} \frac{d_{\mathcal{C}_{1}(\Sigma)}\left(f^{n}(\alpha), \alpha\right)}{n}$ exists and positive, and it is independent of the choice of $\alpha$.

Fix a number $k>1$. We define inductively a pseudo-Anosov mapping class $\Psi_{k} \in \operatorname{Mod}(S)$ as follows. For $m \geq 1$ write $\ell(m)=k^{2^{2 g-2-m}}$. Define 
$\eta_{1}=\phi_{2 g-3}$ and inductively let

$$
\eta_{m}=\phi_{2 g-2-m} \circ \eta_{m-1}^{\ell(m-1)} .
$$

Then for each $m, \eta_{m}$ is a pseudo-Anosov diffeomorphism of $S_{2 g-2-m}$. The mapping class $\Psi_{k}=\eta_{2 g-2}$ is pseudo-Anosov, with train track expansion $\tau$. We refer to Section 6 of 12 for details of this construction.

Now consider the mapping torus $M_{k}$ of $\Psi_{k}$. As a hierarchy for $M_{k}$ is defined by singling out subsurfaces $Y$ of $S$ so that the subsurface projections of the vertical and horizontal measured geodesic laminations of the axis of $\Psi_{k}$ is large, it follows from Proposition 2.2 (and its proof) that $\left(M_{k}\right)_{\text {thick }}$ is $L$-quasi-isometric to an optimal step-homogeneous array of circles of base length $k$ for a constant $L>1$ not depending on $k$.

To give a more detailed account on this fact, the main geodesic of the hierarchy corresponds to a fundamental domain in a quasi-axis in $\mathcal{C}(S)$ for the pseudo-Anosov mapping class $\phi_{0}^{k}$. This main geodesic determines the base circle $L$ of the generalized array of circles in the construction from the proof of Proposition 2.2. The length of this base circle is uniformly equivalent to $k$.

In a second step, the hierarchy contains geodesics in the curve graph of copies of the surface $S_{1}$ whose length is equivalent to $k^{2}$. In the construction of the generalized array of circles, this amounts to blowing up each vertex $v$ of $L$ and replacing it by a single edge and an arc of length equivalent to $k^{2}$ with the same endpoints. Let $G_{1}$ be the resulting graph. Contraction of each vertex arc in $G_{1}$ which consists of a single edge yields an array of circles which is uniformly quasi-isometric to $G_{1}$. This array of circles is uniformly quasi-isometric to an optimal array of circles of depth two. By induction, we conclude that indeed, the thick part of $M_{k}$ is uniformly quasi-isometric to an optimal array of circles of depth $2 g-2$. The proposition follows.

We use Proposition 2.2 and its proof to obtain some additional geometric information on doubly degenerate hyperbolic 3-manifolds which are used in the proof of Theorem 2 .

Let $\gamma \subset \mathcal{T}(S)$ be a bi-infinite Teichmüller geodesic which defines a doubly degenerate hyperbolic 3-manifold $M$ with filling end invariants. Suppose that for some $\varepsilon>0$ the geodesic contains a subarc $\gamma[a, b]$ entirely contained in the $\varepsilon$-thick part $\mathcal{T}(S)_{\varepsilon}$ of $\mathcal{T}(S)$ of all marked hyperbolic surfaces of injectivity radius at least $\varepsilon$. By [24] (see [10] for an explicit statement), there is a number $\chi=\chi(\varepsilon)>0$ so that the map which associates to $t \in[a, b]$ a closed geodesic of smallest length on the hyperbolic surface $\gamma(t)$ is a $\chi$-quasigeodesic in the curve complex $\mathcal{C}(S)$ of $S$. Therefore the endpoints $\gamma(a), \gamma(b)$ define (non-uniquely) a hierarchy $H$ all of whose geodesics different from the main geodesic have uniformly bounded length [25]. Moreover, if $b-a$ is sufficiently large then the length of the main geodesic is larger than any prescribed threshold. 
Let $v$ be any vertex in the main geodesic associated to $\gamma[a, b]$. We are only interested in vertices not too close to the endpoints of the hierarchy $H$. Such a vertex is a multicurve in $S$ whose length becomes short along $\gamma[a, b]$, say at $\gamma(s)$. By Lemma 7.9 of [28], the lengths of the closed geodesics in $M$ in the free homotopy classes of the components of $v$ is uniformly bounded. There is a pleated surface $f: S \rightarrow M$ which maps the curves from a maximal simplex $\Delta \subset \mathcal{C}(S)$ with $v \subset \Delta$ to geodesics in $M$, and these geodesics all have moderate length in $M$. Moreover, the set of simple closed curves on the pleated surface which have uniformly bounded length in $M$ is contained in the $d^{\prime}$-neighborhood of $v$ in $\mathcal{C}(S)$ for some universal number $d^{\prime}>0$.

By the tube penetration Lemma 7.7 of [28], there is a number $r>0$ only depending on $\varepsilon$ and $g$ with the following property. Let $s \in[a+r, b-r]$ and let $v \in H$ be a vertex corresponding to a multicurve which becomes short for $\gamma(s)$; then the diameter of a pleated surface mapping $v$ geodesically is uniformly bounded.

Theorem 6.2 of [4 now shows that up to enlarging $r$, such a pleated surface $f:(S, \sigma) \rightarrow M$ can be deformed with a homotopy to an embedding $F:(S, \sigma) \rightarrow M$ with the following properties.

(1) $F(S)$ is contained in the $r$-neighborhood of $f(S)$.

(2) The second derivatives of $F$ are uniformly bounded.

We use this to show

Proposition 2.6. For every $\varepsilon>0$ there exists a constant $c_{2}=c_{2}(g, \varepsilon)>0$ with the following property. Let $\hat{M}$ be a doubly degenerate hyperbolic 3manifold which is an infinite cyclic cover of a mapping torus $M$ of genus g. Suppose that the Teichmüller geodesic $\gamma$ defining $M$ contains a segment $\gamma[a, b] \subset \mathcal{T}(S)_{\varepsilon}$ of length $b-a \geq 2 c_{2}$. Then $\hat{M}$ contains a smooth embedded 3-manifold $N_{0}$ with boundary $\partial N_{0}$ with the following properties.

(1) $\partial N_{0}=\Sigma_{a} \cup \Sigma_{b}$, and there are diffeomorphisms $f_{1}:\left(S, \gamma\left(a+c_{2}\right)\right) \rightarrow$ $\Sigma_{a}, f_{2}:\left(S, \gamma\left(b-c_{2}\right)\right) \rightarrow \Sigma_{b}$ whose derivatives are uniformly bounded.

(2) There is a smooth surjective map $N_{0} \rightarrow[a, b]$ of uniformly bounded derivatives.

If $\gamma\left[a^{\prime}, b^{\prime}\right] \subset \mathcal{T}(S)_{\varepsilon}$ is another such segment so that $[a, b] \cap\left[a^{\prime}, b^{\prime}\right]=\emptyset$ then the corresponding 3-manifolds $N_{0}, N_{0}^{\prime}$ are disjoint.

Proof. The above discussion implies that $M$ contains a submanifold which is a union of pieces diffeomorphic to $S \times[0,1]$ whose boundaries are deformations of pleated surfaces determined by hyperbolic metrics along $\gamma[a, b]$. The number of these pieces is proportional to $b-a$. Disjoint intervals in the parameter space of $\gamma$ as in the proposition give rise to disjoint pieces.

The pieces are of uniformly bounded diameter, and their boundary surfaces have uniformly bounded geometry. Then there is a controlled tubular neighborhood of each of these boundary surfaces, and these neighborhoods can be used to construct the map onto $[a, b]$. 


\section{Arrays of CIRCles}

The main result of [22] states the following. Let $M$ be a closed Riemannian manifold of bounded geometry whose injectivity radius is bounded from below by a fixed positive constant. If $M$ is uniformly quasi-isometric to a finite graph $G$ then the smallest positive eigenvalue of $M$ is uniformly equivalent to the smallest positive eigenvalue of $G$. This statement is also valid without modification for compact manifolds $M$ with boundary and Neumann boundary conditions (see [19] for a more precise statement).

Let us consider as before a hyperbolic mapping torus $M$ of genus $g \geq$ 2. We showed in Section 2 that the thick part $M_{\text {thick }}$ of $M$ is $L=L(g)$ quasi-isometric to a generalized array of circles of depth at most $2 g-2$. Thus to estimate the smallest eigenvalue of $M_{\text {thick }}$ with Neumann boundary conditions it suffices to estimate the smallest eigenvalue of a generalized array of circles of a given depth. The purpose of this section is to establish such an estimate.

Let for the moment $G$ be any finite connected graph with vertex set $\mathcal{V}(G)$ and edge set $\mathcal{E}(G)$. Denote by $\mathcal{F}_{0}(G)$ the vector space of functions

$$
f: \mathcal{V}(G) \rightarrow \mathbb{R}
$$

with the property that $\sum_{v} f(v)=0$. We equip $\mathcal{F}_{0}(G)$ with the usual $\ell^{2}$-inner product

$$
(f, h)=\sum_{v} f(v) g(v) .
$$

For each such function $f$, the Rayleigh quotient $\mathcal{R}(f)$ is defined by

$$
\mathcal{R}(f)=\frac{\sum_{v} \sum_{w \sim v}(f(w)-f(v))^{2} / p(v)}{\sum_{v} f^{2}(v)}
$$

where $p(v)$ is the degree of the vertex $v$, and $v \sim w$ means that $v$ and $w$ are connected by an edge. The first eigenvalue of $G$ is defined as

$$
\lambda_{1}(G)=\inf \left\{\mathcal{R}(f) \mid 0 \neq f \in \mathcal{F}_{0}(G)\right\} .
$$

In the sequel we adopt analytic notations, and we write

$$
\int\left(f^{\prime}\right)^{2}=\sum_{v} \sum_{w \sim v}(f(w)-f(v))^{2} / p(v)
$$

and $\int f^{2}=\sum_{v} f^{2}(v)$.

Throughout the rest of this section we view a graph $G$ as a metric space with edges of length one. Thus the length of a subarc of $G$ equals its combinatorial length, i.e. the number of its edges.

Our first goal is to establish an upper bound for the first eigenvalue of an array of circles. In a second step, we then extend the bound to a generalized array.

We will make use of the Minmax-principle which is equally valid for the Laplacian on manifolds as well as for the Laplacian on graphs. For a finite graph $G$ it states the following. 
Let $\rho_{0}, \rho_{1}: \mathcal{V}(G) \rightarrow \mathbb{R}$ be any two nontrivial functions with disjoint support; then

$$
\lambda_{1}(G) \leq \max _{i=0,1} \mathcal{R}\left(\rho_{i}\right) .
$$

Proposition 3.1. Let $G$ be an array of circles of depth $h$; then

$$
\lambda_{1}(G) \leq 64 \pi^{2} / \operatorname{vol}(G)^{2^{h} /\left(2^{h}-1\right)} .
$$

Proof. We show by induction on $h$ the following. Let $G$ be an array of circles of depth $h$; then for every vertex $v$ of the base circle of $G$ there is a function $f \in \mathcal{F}_{0}(G)$ with $f(v)=0$ so that $\mathcal{R}(f) \leq 64 \pi^{2} / \operatorname{vol}(G)^{2^{h} /\left(2^{h}-1\right)}$.

In the case $h=1, G$ is a circle and the claim is straighforward. Thus assume that the claim holds true for $h-1$. Let $G$ be an array of circles of depth $h$, with base circle $L$. Assume first that there is a vertex $v$ of $L$ so that the volume of the descendant $G_{v}$ of $L$ at $v$ (i.e. the array of circles of depth at most $h-1$ attached to $L$ at $v)$ is at least $\operatorname{vol}(G)^{\left(2^{h}-2\right) /\left(2^{h}-1\right)}$.

By the induction hypothesis, there is a function $f \in \mathcal{F}_{0}\left(G_{v}\right)$ with $f(v)=0$ and such that

$$
\mathcal{R}(f) \leq 64 \pi^{2} / \operatorname{vol}\left(G_{v}\right)^{2^{h-1} /\left(2^{h-1}-1\right)} .
$$

Extend $f$ by zero to $G$. The extended function $F$ vanishes on the base circle $L$ of $G$, and it is contained in $\mathcal{F}_{0}(G)$. Moreover,

$$
\begin{aligned}
\mathcal{R}(F) & \leq 64 \pi^{2} /\left(\operatorname{vol}(G)^{\left(2^{h}-2\right) /\left(2^{h}-1\right)}\right)^{2^{h-1} /\left(2^{h-1}-1\right)} \\
& =64 \pi^{2} / \operatorname{vol}(G)^{2^{h} /\left(2^{h}-1\right)} .
\end{aligned}
$$

Thus the function $F$ satisfies all the requirements in the above claim, for every vertex of the base circle.

The second case is that the volume of every descendant of $L$ is strictly smaller than

$$
\operatorname{vol}(G)^{\left(2^{h}-2\right) /\left(2^{h}-1\right)}=E .
$$

Let $\ell \geq 2$ be the length of the base circle $L$, let $v \in L$ be any vertex and let $\alpha:[0, \ell] \rightarrow L$ be a simplicial parametrization of $L$ by arc length with $\alpha(0)=v$ which maps the integral points in $[0, \ell]$ to the vertices of $L$. For $1 \leq k \leq \ell$ and $t \in[k-1 / 2, k+1 / 2)$, let $\eta(t)$ be one plus the volume $\operatorname{vol}\left(G_{\alpha(k)}\right)$ of the descendant $G_{\alpha(k)}$ of $L$ at $\alpha(k)$ (with the obvious interpretation for $k=\ell$ ). Note that $1 \leq \eta(t) \leq E$ for all $t$.

Define

$$
\beta(t)=\int_{0}^{t} \frac{1}{E} \eta(s) d s
$$

then $\beta$ is differentiable outside the points $k+\frac{1}{2}$ for $k \in \mathbb{Z}$, and moreover $0<\beta^{\prime}(t) \leq 1$ for all $t$. More precisely, $\beta$ is a piecewise-linear continuous function which is strictly increasing, and

$$
\beta(\ell)=(\ell+\operatorname{vol}(G)) / E=\ell / E+E^{1 /\left(2^{h}-2\right)}=E^{\prime} .
$$


Let $m \in(0, \ell)$ be such that $\beta(m)=E^{\prime} / 2$. For $t \in[0, \ell]$ define a function $f_{1}$ supported in $[0, m]$ by

$$
f_{1}(t)= \begin{cases}\sin \left(4 \pi \beta(t) / E^{\prime}\right) & \text { if } 0 \leq t \leq m \\ 0 & \text { otherwise }\end{cases}
$$

and define similarly a function $f_{2}$ supported in $[m, \ell]$.

By the mean value theorem, for each $k$ there exists some $t_{k} \in\left[k-\frac{1}{2}, k+\frac{1}{2}\right]$ such that $f_{i}\left(t_{k}\right)$ satisfies

$$
f_{i}\left(t_{k}\right)^{2}=\int_{k-\frac{1}{2}}^{k+\frac{1}{2}} f_{i}^{2}(s) d s
$$

Define

$$
F_{i}(\alpha(k))=f_{i}\left(t_{k}\right)
$$

and extend $F_{i}$ to a function on $G$ which is constant on each of the arrays of circles $G_{\alpha(i)}$ which are attached to the vertices of the circle $L$. Since the function $\eta$ is constant on each of the intervals $\left[k-\frac{1}{2}, k+\frac{1}{2}\right]$, we conclude that

$$
\int_{0}^{\ell} f_{i}^{2}(t) \eta(t) d t=\sum_{v} F_{i}^{2}(v)
$$

Our strategy now is to show that $\mathcal{R}\left(F_{i}\right)$ is close to the quotient

$$
\mathcal{R}\left(f_{i}\right)=\int_{0}^{\ell}\left(f_{i}^{\prime}\right)^{2} d t / \int_{0}^{\ell} f_{i}^{2}(t) \eta(t) d t
$$

and furthermore estimate $\mathcal{R}\left(f_{i}\right)$. The above claim then follows from the Minmax theorem, applied to the functions $F_{1}$ and $F_{2}$ (whose mean may not be zero). Namely, with a small modification of the initial functions $f_{i}$ we may assure that $F_{1}(v)=F_{2}(v)=0$, and we can find a function in $\mathcal{F}_{0}(G)$ which vanishes at $v$, with controlled Rayleigh quotient, as a linear combination of $F_{1}$ and $F_{2}$.

To show that $\mathcal{R}\left(F_{i}\right)$ is close to $\mathcal{R}\left(f_{i}\right)$, by equation (4) it suffices to compare $\sum_{v} \sum_{w \sim v}\left(F_{i}(w)-F_{i}(v)\right)^{2} / p(v)$ to $\int_{0}^{\ell}\left(f_{i}^{\prime}\right)^{2}(t) d t$. We carry this estimate out for $f=f_{1}$ and $F=F_{1}$, the calculation for $f_{2}$ and $F_{2}$ is identical.

By the definition of an array of circles, the valency $p(v)$ of every vertex $v$ of the base circle equals 2 or 4 . For each $v_{j}=\alpha(j)$ we have

$$
\sum_{w \sim v_{j}}\left(F(w)-F\left(v_{j}\right)\right)^{2}=\left(F\left(v_{j-1}\right)-F\left(v_{j}\right)\right)^{2}+\left(F\left(v_{j+1}\right)-F\left(v_{j}\right)\right)^{2} .
$$


Thus

$$
\begin{aligned}
& \sum_{v} \sum_{w \sim v}(F(v)-F(w))^{2} / p(v) \\
\leq & \frac{1}{2} \sum_{k=1}^{\ell}\left(\left(F\left(v_{k-1}\right)-F\left(v_{k}\right)\right)^{2}+\left(F\left(v_{k+1}\right)-F\left(v_{k}\right)\right)^{2}\right) \\
= & \sum_{k=1}^{\ell}\left(F\left(v_{k}\right)-F\left(v_{k-1}\right)\right)^{2} .
\end{aligned}
$$

Recall that there is some $t_{j} \in[j-1 / 2, j+1 / 2]$ so that $F\left(v_{j}\right)=f\left(t_{j}\right)$. Since $\left|t_{j+1}-t_{j}\right| \leq 2$, the Cauchy Schwarz inequality yields

$$
\left(F\left(v_{j+1}\right)-F\left(v_{j}\right)\right)^{2}=\left(\int_{t_{j-1}}^{t_{j}} f^{\prime}(t) d t\right)^{2} \leq 4 \int_{t_{j-1}}^{t_{j}}\left(f^{\prime}(t)\right)^{2} d t .
$$

Together this implies the estimate

$$
\sum_{v} \sum_{w \sim v}(F(w)-F(v))^{2} / p(v) \leq 2 \int_{0}^{\ell}\left(f^{\prime}(t)\right)^{2} d t .
$$

As a consequence, we obtain $\mathcal{R}(F) \leq 4 \mathcal{R}(f)$ as desired.

For the estimate of $\mathcal{R}(f)$ (here as before, $f=f_{1}$ ) recall that $0<\beta^{\prime}(t) \leq 1$ and $f^{\prime}(t)=4 \pi \cos \left(4 \pi \beta(t) / E^{\prime}\right) \beta^{\prime}(t) / E^{\prime}$. Therefore

$$
\left(f^{\prime}(t)\right)^{2}=\frac{16 \pi^{2}}{\left(E^{\prime}\right)^{2}} \cos \left(4 \pi \beta(t) / E^{\prime}\right)^{2}\left(\beta^{\prime}(t)\right)^{2} \leq \frac{16 \pi^{2}}{\left(E^{\prime}\right)^{2}} \cos \left(4 \pi \beta(t) / E^{\prime}\right)^{2} \beta^{\prime}(t) .
$$

This implies

$$
\begin{aligned}
\int_{0}^{\ell}\left(f^{\prime}(t)\right)^{2} d t & \leq \frac{16 \pi^{2}}{\left(E^{\prime}\right)^{2}} \int_{0}^{m} \cos \left(4 \pi \beta(t) / E^{\prime}\right)^{2} \beta^{\prime}(t) d t \\
& =\frac{16 \pi^{2}}{\left(E^{\prime}\right)^{2}} \int_{0}^{E^{\prime} / 2} \cos \left(4 \pi s / E^{\prime}\right)^{2} d s
\end{aligned}
$$

With the same argument, using $\eta(t)=E \beta^{\prime}(t)$, we obtain

$$
\int_{0}^{m} f^{2}(t) \eta(t) d t=E \int_{0}^{E^{\prime} / 2} \sin \left(4 \pi s / E^{\prime}\right)^{2} d s .
$$

Since $\int_{0}^{E^{\prime} / 2} \cos \left(4 \pi s / E^{\prime}\right)^{2} d s=\int_{0}^{E^{\prime} / 2} \sin \left(4 \pi s / E^{\prime}\right)^{2} d s$ and $E^{\prime} \geq E^{1 /\left(2^{h}-2\right)}$, we deduce

$$
\mathcal{R}(f) \leq 16 \pi^{2} E^{-1}\left(E^{\prime}\right)^{-2} \leq 16 \pi^{2} / E^{2^{h} /\left(2^{h}-2\right)}=16 \pi^{2} / \operatorname{vol}(G)^{2^{h} /\left(2^{h}-1\right)} .
$$

This is what we wanted to show.

Our next goal is to extend Proposition 3.1 to generalized arrays of circles.

Proposition 3.2. Let $G$ be a generalized array of circles of depth at most $h$; then

$$
\lambda_{1}(G) \leq 256 \pi^{2} h^{h-1} / 3\left(\operatorname{vol}(G)^{2^{h} /\left(2^{h}-1\right)}\right) .
$$


Proof. We prove the proposition by constructing for every generalized array of circles $G$ of depth $h$ an array of circles $H$ of depth at most $h$, and a continuous simplicial surjective map $\Psi: G \rightarrow H$. This construction is done in such a way that

- $\lambda_{1}(G) \leq \frac{4}{3} \lambda_{1}(H)$,

- $\operatorname{vol}(H) \geq h^{-h+1} \operatorname{vol}(G)$.

Then from Proposition 3.1 we have

$$
\begin{aligned}
\lambda_{1}(G) \leq \frac{4}{3} \lambda_{1}(H) & \leq \frac{4}{3} 64 \pi^{2} / \operatorname{vol}(H)^{2^{h} /\left(2^{h}-1\right)} \\
& \leq \frac{256 \pi^{2}}{3} /\left(h^{-h+1} \operatorname{vol}(G)\right)^{2^{h} /\left(2^{h}-1\right)}
\end{aligned}
$$

which is what we wanted to show.

For the construction of $H$, note that by the inductive definition, a generalized array of circles of depth at most $h$ differs from an array of circles by allowing the blow-up of vertices. Recall that this means that we start with a base circle $L$, and for each vertex $v$ of $L$, we allow to either attach to $v$ a generalized array of circles of depth at most $h-1$, or to replace $v$ by $s \leq h \operatorname{arcs} a_{1}, \ldots, a_{s}$. In the second case, to each such arc $a_{i}$ is associated a positive weight $m_{i} \geq 1$ so that $h \geq \sum_{i} m_{i}$. To each interior vertex of the arc $a_{i}$ there is attached a (possibly trivial) generalized array of circles of depth at most $m_{i}-1$, allowing blow-ups of vertices as before. Let $v_{1}, v_{2}$ be the common endpoints of the $\operatorname{arcs} a_{i}$. Define the mass of $a_{i}$ to be the total volume of the connected component $E\left(a_{i}\right)$ of $G-\left\{v_{1}, v_{2}\right\}$ containing the interior of the $\operatorname{arc} a_{i}$.

The construction of the array of circles $H$ is carried out inductively with the following algorithm. Begin with the base circle $L$ of $G$. If no vertex of $L$ is blown up in $G$ in the inductive build-up of $G$ then repeat the construction with all circles in $G$ of depth two. Otherwise let $v_{1}, \ldots, v_{s}$ be the vertices of $L$ which are blown-up in $G$. For each $i \leq s$, choose a vertex arc $a_{i}$ for the vertex $v_{i}$ with the largest mass. Define $G_{1}$ to be the graph obtained from $G$ by collapsing each of the graphs $E\left(b_{j}\right)$ for all vertex arcs $b_{j} \neq a_{i}$ for the vertex $v_{i}$ to a point. This modification identifies the endpoints of the arc $a_{i}$. Or, equivalently, in $G_{1}$, the arc $a_{i}$ is replaced by a circle of the same length.

Since the sum of the masses of the vertex arcs for the vertex $v_{i}$ is not bigger than $h$ times the mass of $a_{i}$, the volume of $G_{1}$ is not smaller than $\operatorname{vol}(G) / h$. Moreover, $G_{1}$ is a generalized array of circles with no blown-up vertex on the base circle. Note that there is a natural surjective simplicial map $\Psi_{1}: G \rightarrow G_{1}$ which for each $i$ maps the graph $E\left(a_{i}\right)$ isomorphically, and it maps the blown-up base circle in the construction of $G$ to the base circle in $G_{1}$.

Repeat this construction with $G_{1}$ and the blown-up circles of depth two. Since no vertex of the circles of depth $h$ is blown up, in at most $h-1$ such steps we construct in this way an array of circles $H$ with $\operatorname{vol}(H) \geq$ $h^{-h+1} \operatorname{vol}(G)$. There is a natural simplicial surjection $\Psi: G \rightarrow H$. 
Let now $f \in \mathcal{F}_{0}(H)$ be any function. We show next that $\int\left((\Psi \circ f)^{\prime}\right)^{2} \leq$ $\frac{4}{3} \int\left(f^{\prime}\right)^{2}$. By definition,

$$
\int\left((f \circ \Psi)^{\prime}\right)^{2}=\sum_{v} \frac{1}{p(v)} \sum_{w \sim v}(f \circ \Psi(v)-f \circ \Psi(w))^{2} .
$$

Note that $\sum_{w \sim v}(f \circ \Psi(v)-f \circ \Psi(w))^{2}=0$ if $v$ is an interior vertex of an arc collapsed by $\Psi$. If $v_{1}, v_{2}$ are the two endpoints of such an arc, and if $v=\Psi\left(v_{1}\right)=\Psi\left(v_{2}\right)$ then

$$
\begin{aligned}
\sum_{w \sim v_{1} \in G}\left(f \circ \Psi\left(v_{1}\right)-f \circ \Psi(w)\right)^{2} & +\sum_{w \sim v_{2} \in G}\left(f \circ \Psi\left(v_{2}\right)-f \circ \Psi(w)\right)^{2} \\
& =\sum_{w \sim v \in H}(f(v)-f(w))^{2} .
\end{aligned}
$$

Also note that $p\left(v_{1}\right)=p\left(v_{2}\right) \geq 3$ while $p(v)=4$. Hence,

$$
\sum_{i=1,2} \sum_{w \sim v_{i} \in G}\left(f \circ \Psi\left(v_{i}\right)-f \circ \Psi(w)\right)^{2} / p\left(v_{i}\right) \leq \frac{4}{3} \sum_{w \sim v \in H}(f(v)-f(w))^{2} / p(v) .
$$

For all other vertices, both denominator and numerator coincide when we switch from $f \circ \Psi$ to $f$. Thus we have

$$
\int_{G}\left((f \circ \Psi)^{\prime}\right)^{2} \leq \frac{4}{3} \int_{H}\left(f^{\prime}\right)^{2}
$$

as claimed.

The function $f \circ \Psi$ need not be contained in $\mathcal{F}_{0}(G)$. Let $m=\int f \circ \Psi$ and let $\hat{f}=f \circ \Psi-m$. Then $\hat{f} \in \mathcal{F}_{0}(G)$, and $\int\left(\hat{f}^{\prime}\right)^{2}=\int\left((f \circ \Psi)^{\prime}\right)^{2}$. Hence using the estimate (7), for the purpose of the proposition it suffices to show that $\int \hat{f}^{2} \geq \int f^{2}$.

To this end note that there is a subset of the set of vertices of $G$, say the set $\mathcal{V}_{1}$, which is mapped by $\Psi$ bijectively onto the set of vertices of $H$ : For vertex $\operatorname{arcs} a_{1}, \ldots, a_{s}$ of a blown-up vertex $v$, with endpoints $v_{1}, v_{2}$, choose either $v_{1}$ or $v_{2}$ to be in $\mathcal{V}_{1}$ and declare the second endpoint as well as all interior vertices of any erased $\operatorname{arc} a$ and all vertices of any of the subgraphs of $G$ which are attached to interior points of $a$ to be in $\mathcal{V}(G)-\mathcal{V}_{1}$. Proceed by induction.

Now $f \in \mathcal{F}_{0}(H)$ and therefore $\int_{H} f m=0$. This implies that

$$
\int_{G} \hat{f}^{2} \geq \sum_{v \in \mathcal{V}_{1}}(f \circ \Psi-m)(v)^{2}=\int_{H}(f-m)^{2}=\int_{H}\left(f^{2}+m^{2}\right) \geq \int_{H} f^{2}
$$

which is what we wanted to show.

We are left with finding examples of graphs which realize the bounds in Proposition 3.1 up to a universal constant. To this end we say that the support $\operatorname{supp}\left(f^{\prime}\right)$ of the derivative of $f$ consists of all edges $e$ in $G$ so that the values of $f$ at the endpoints of $e$ do not coincide.

We begin with the following elementary 
Lemma 3.3. Let $G$ be any finite connected graph. Assume that there is a decomposition $\mathcal{F}_{0}(G)=A \oplus B$ which is orthogonal for the $\ell^{2}$-inner product. Assume furthermore that the supports of the derivatives of functions in $A, B$ are disjoint; then

$$
\lambda_{1}(G)=\min \left\{\lambda_{1}(A), \lambda_{1}(B)\right\}
$$

where $\lambda_{1}(A)$ (or $\lambda_{1}(B)$ ) is the infimum of the Rayleigh quotients over all functions of the space $A$ (or $B$ ).

Proof. Under the assumption of the lemma, if $\phi \in \mathcal{F}_{0}(G)$ is arbitrary then $\phi=\alpha+\beta$ for some $\alpha \in A, \beta \in B$. Since the supports of the derivatives of functions in $A, B$ are disjoint, formula (2) implies that

$$
\int\left(\phi^{\prime}\right)^{2}=\int\left(\alpha^{\prime}\right)^{2}+\int\left(\beta^{\prime}\right)^{2} \text {. }
$$

Now if $s=\min \left\{\lambda_{1}(A), \lambda_{1}(B)\right\}$ then

$$
\int\left(\alpha^{\prime}\right)^{2} \geq s \int \alpha^{2}, \int\left(\beta^{\prime}\right)^{2} \geq s \int \beta^{2}
$$

and consequently

$$
\int\left(\phi^{\prime}\right)^{2}=\int\left(\alpha^{\prime}\right)^{2}+\left(\beta^{\prime}\right)^{2} \geq s \int\left(\alpha^{2}+\beta^{2}\right)=s \int(\alpha+\beta)^{2}=s \int \phi^{2}
$$

where the second last equality follows from the assumption that $\alpha, \beta$ are orthogonal for the $\ell^{2}$-inner product. This shows the lemma.

Recall from Section 2 the definition of a step-homogeneous array of circles. Such an array $G$ is characterized by the property that all circles of depth $j$ have the same length $\ell(j)$. The array of circles is called optimal if there exists a number $k \geq 3$ so that $\ell(j)=k^{2^{j-1}}$.

Proposition 3.4. For every $h \geq 1$ there is a number $q=q(h)>0$ with the following property. Let $G$ be an optimal step-homogeneous array of circles of depth $h$; then

$$
\lambda_{1}(G) \geq q / \operatorname{vol}(G)^{2^{h} /\left(2^{h}-1\right)} .
$$

Proof. Let for the moment $G$ be an arbitrary step homogeneous array of circles of depth $h$. Then for every $m \leq h$, the union $G_{m}$ of all circles in $G$ of depth at most $m$ is a step homogeneous array of circles of depth $m$. However, if $1 \leq m \leq h-2$ then the closure in $G$ of a component of $G-G_{m}$ is an array of circles which is not step homogeneous. Namely, its base circle contains a distinguished vertex (the attaching vertex) of valency two. Deleting this vertex results in a step homogeneous array of circles.

Write $A \asymp B$ if $n^{-1} B \leq A \leq n B$ for a universal constant $n>0$, and write $A \preceq B$ if $A \leq n B$ for a universal constant $n>0$.

Let as before $\ell(j) \geq 3$ be the length of a circle in $G$ of depth $j$. The volume of $G$ can recursively be computed by

$$
\operatorname{vol}\left(G_{m}\right)=\ell(m) \chi(m-1) \operatorname{vol}\left(G_{m-1}\right)
$$


where $\chi(m-1)$ is the number of bivalent vertices in $G_{m-1}$ (which is just $\left.\left|\mathcal{V}\left(G_{m-1}\right)\right|-\left|\mathcal{V}\left(G_{m-2}\right)\right|\right)$. This implies the estimate $\operatorname{vol}(G) \asymp \prod_{j=1}^{h} \ell(j)$. Thus if $G$ is optimal, with base circle of length $k \geq 3$, then $\operatorname{vol}(G) \asymp k^{2^{h}-1}$. Our goal is to show that

$$
\lambda_{1}(G) \succeq 1 / k^{2^{h}} .
$$

When $h=1, G$ is a circle of length $k$, and the estimate $\lambda_{1}(G) \geq 4 / k^{2}$ is an easy consequence of the following. Any function $F$ on the vertex set of $G$ can be extended by convex combination to a continuous piecewise affine function $f$ on all of $G$. If $\sum_{v} F(v)=0$ then $\int_{G} f=0$ where integration is with respect to the standard Lebesgue measure which gives an edge the volume one. The Rayleigh quotients can be compared by $\mathcal{R}(F) \geq \frac{1}{2} \mathcal{R}(f)$. Now the smallest non-zero eigenvalue of a smooth circle of length $R$ equals $4 \pi^{2} / R^{2}$ which yields the required estimate for $\lambda_{1}(G)$.

Furthermore, let $f$ be any function on $\mathcal{V}(G)$ which either vanishes at a vertex $v$ or changes signs at $v$ (by this we mean that $f$ assumes a value of opposite sign at a neighbor of $v$ ). Cut $G$ open at $v$, glue two copies of the cut open arc to a circle $\hat{G}$ of double length and extend $f$ to a function $F$ on $\mathcal{V}(\hat{G})$ by reflection at the two copies of $v$ in $\hat{G}$ (with the obvious interpretation if $f$ changes signs at $v)$. Then $\sum_{w} F(w)=0$, and the Rayleigh quotients $\mathcal{R}(F)$ and $\mathcal{R}(f)$ can be compared as follows.

If $f(v)=0$ then for the two copies $v_{1}, v_{2}$ of $v$ in $G$, we have

$$
\sum_{i} \sum_{w \sim v_{i}}\left(F(w)-F\left(v_{i}\right)\right)^{2}=2 \sum_{w \sim v}(f(w)-f(v))^{2}
$$

and similarly for the other vertices of $G$ and their two preimages in $\hat{G}$, and consequently $\mathcal{R}(F)=\mathcal{R}(f)$.

Now assume that $f$ changes sign at $v$. Let $w_{1}, w_{2}$ be the two neighbors of $v$ and assume that the signs of $f(v)$ and $f\left(w_{1}\right)$ are opposite. The contribution of the two preimages $v_{1}, v_{2}$ of $v$ in $\hat{G}$ in the expression for $\int\left(F^{\prime}\right)^{2}$ equals

$\left(-f\left(w_{1}\right)-f(v)\right)^{2}+\left(f\left(w_{1}\right)-f(v)\right)^{2}+\left(-f\left(w_{2}\right)-f(v)\right)^{2}+\left(f\left(w_{2}\right)-f(v)\right)^{2}$.

Now if the signs of $f\left(w_{1}\right)$ and $f\left(w_{2}\right)$ coincide then $\left(-f\left(w_{i}\right)-f(v)\right)^{2} \leq$ $\left(f\left(w_{i}\right)-f(v)\right)^{2}$ for $i=1,2$ and hence $\mathcal{R}(F) \leq \mathcal{R}(f)$. Otherwise note that

$$
\begin{aligned}
\left(-f\left(w_{2}\right)-f(v)\right)^{2} & \leq 2\left(\left(-f\left(w_{2}\right)-f\left(w_{1}\right)\right)^{2}+\left(f\left(w_{1}\right)-f(v)\right)^{2}\right) \\
& \leq 4\left(f\left(w_{2}\right)-f(v)\right)^{2}+6\left(f\left(w_{1}\right)-f(v)\right)^{2}
\end{aligned}
$$

which implies that $\mathcal{R}(F) \leq 3 \mathcal{R}(f)$. Thus the Rayleigh quotient of the function $f$ is not smaller than $\frac{1}{3} \lambda_{1}(\hat{G}) \geq 1 / 3 k^{2}$.

We now proceed by induction on $h$; then case $h=1$ was treated above. Thus assume that the estimate (8) holds true for optimal step-homogeneous arrays of depth at most $h-1 \geq 1$ and let $G$ be an optimal step homogeneous array of depth $h$, with base circle of length $k$. 
Our strategy is to apply Lemma 3.3 to the subspace of $\mathcal{F}_{0}(G)$ of functions which are constant on each of the circles of depth $h$ and compare their Rayleigh quotients to $\lambda_{1}\left(G_{h-1}\right)$. The following construction is used to circumvent the difficulty that a circle of depth $h$ in $G$ is attached to the vertices of $G_{h-1}$ of valence two but not to every vertex. We construct from $G$ a graph $H$ of uniformly bounded valency which is uniformly quasi-isometric to $G$ and which does not have this problem. We then use Theorem 2.1 of [22] to compare $\lambda_{1}(G)$ to $\lambda_{1}(H)$.

The graph $H$ is constructed successively as follows. If $h \leq 2$ then put $H=G$. Otherwise for each vertex $v \in G_{h-2}-G_{h-3}$, collapse one of the two edges in $G_{h-1}-G_{h-2}$ which are incident on $v$ to a point. Let $\hat{G}$ be the resulting graph. It arises from a graph of valency four by merging pairs of vertices, with any vertex involved in at most one such process. Thus the valency of $\hat{G}$ is at most 7 , and the collapsing map $\hat{\Psi}: G \rightarrow \hat{G}$ is a oneLipschitz 2-quasi-isometry which maps $G_{h-3}$ isomorphically. Note that $\hat{G}$ is obtained from $\hat{\Psi}\left(G_{h-1}\right)$ by attaching to each vertex of $\hat{\Psi}\left(G_{h-1}-G_{h-3}\right)$ a circle of length $k^{2^{h-1}}$.

Repeat this construction with the subgraph $\hat{\Psi}\left(G_{h-3}\right)$ of $\hat{G}$, now collapsing edges in $\hat{\Psi}\left(G_{h-2}-G_{h-3}\right)$. In $h-2$ such steps we obtain a graph $H$ and a surjective simplicial projection $\Psi: G \rightarrow H$ with the following properties.

(1) The valency of $H$ is at most $4 h$.

(2) $\Psi$ is an $m$-quasi-isometry for a number $m=m(h) \geq 2$ only depending on $h$ but not on $k$.

(3) $Q=\Psi\left(G_{h-1}\right)$ is $m$-quasi-isometric to $G_{h-1}$.

(4) $H$ is obtained from $Q$ by attaching to each vertex $v$ of $Q$ a circle $H_{v}$ of length $k^{2^{h-1}}$.

By Theorem 2.1 of [22] (note that Mantuano uses the notion rough isometry for our more standard terminology quasi-isometry) and properties (1) and (2) above, it now suffices to show the existence of a number $q=q(h)>0$ so that $\lambda_{1}(H) \geq q / k^{2^{h}}$. By property (3) above, by the induction hypothesis and by Theorem 2.1 of [22], applied to $G_{h-1}$ and its image $Q$ under the map $\Psi$, we may assume that $\lambda_{1}(Q) \geq q^{\prime} / k^{2^{h-1}}$ for a number $q^{\prime}>0$ only depending on $h-1$ but not on $k$.

Let $D \subset \mathcal{F}_{0}(H)$ be the linear subspace of all functions on $\mathcal{V}(H)$ which are constant on the circles $H_{v}$ for all vertices $v$ of $Q$. Let $E \subset \mathcal{F}_{0}(H)$ be the linear subspace of functions which are constant on $Q$. By definition, the supports of the derivatives of any two functions $d \in D, e \in E$ are disjoint.

We claim that $\mathcal{F}_{0}(H)=D \oplus E$. To this end let $f \in \mathcal{F}_{0}(H)$ and let $\hat{f}$ be the unique function on $\mathcal{V}(H)$ which coincides with $f$ on $Q \subset H$ and is constant on each graph $H_{v} \subset H$ for every $v \in \mathcal{V}(Q)$. Let $a=\sum_{w \in H} \hat{f}(w)$ and define

$$
\Pi(f)=\hat{f}-a /|\mathcal{V}(H)|
$$


Then $\Pi: f \in \mathcal{F}_{0}(H) \rightarrow \Pi(f) \in D$ is a linear projection, i.e. $\Pi$ is linear, maps $\mathcal{F}_{0}(H)$ into $D$ and equals the identity on the subspace $D$ of $\mathcal{F}_{0}(H)$. Similarly, Id $-\Pi: \mathcal{F}_{0}(H) \rightarrow E$ is a linear projection as well.

The subspaces $D, E$ of $\mathcal{F}_{0}(H)$ are not orthogonal for the $\ell^{2}$-inner product, but as $\int\left(\alpha^{2}+\beta^{2}\right) \geq \frac{1}{2} \int(\alpha+\beta)^{2}$ for any two functions $\alpha, \beta$ on $H$, Lemma 3.3 and its proof implies that

$$
\lambda_{1}(H) \geq \frac{1}{2} \min \left\{\lambda_{1}(D), \lambda_{1}(E)\right\} .
$$

Our strategy now is to estimate $\lambda_{1}(D)$ and $\lambda_{1}(E)$ separately. We begin with estimating $\lambda_{1}(D)$.

Thus let $d \in D$ and let $d_{Q}$ be the restriction of $d$ to $Q$. By the definition of $D$, we have $\operatorname{supp}\left(d^{\prime}\right) \subset Q$. The degree of a vertex $v \in Q \subset H$ viewed as a vertex in $H$ is at most twice its degree as a vertex in $Q$ and therefore

$$
\int\left(d_{Q}^{\prime}\right)^{2} \leq 2 \int\left(d^{\prime}\right)^{2}
$$

Since for every vertex $v \in Q$ the function $d$ is constant on the circle $H_{v}$ and such a circle has precisely $k^{2^{h-1}}$ vertices, we conclude that

$$
\sum_{w \in H_{v}} d^{2}(w)=k^{2^{h-1}} d_{Q}^{2}(v)
$$

and hence

$$
k^{2^{h-1}} \int d_{Q}^{2}=\int d^{2}
$$

The estimates (910) imply that

$$
\mathcal{R}(d) \geq \mathcal{R}\left(d_{Q}\right) / 2 k^{2^{h-1}} .
$$

On the other hand, we also have

$$
\int d=k^{2^{h-1}} \int d_{Q}
$$

and therefore $d_{Q} \in \mathcal{F}_{0}(Q)$ and hence $\mathcal{R}\left(d_{Q}\right) \geq \lambda_{1}(Q)$. Thus by the induction hypothesis, we obtain

$$
\lambda_{1}(D) \geq q^{\prime} / 2 k^{2^{h-1}} k^{2^{h-1}}=q / k^{2^{h}}
$$

where $q=q^{\prime} / 2$ only depends on $h$.

We are left with estimating $\lambda_{1}(E)$. To this end define another graph $W$ as follows. The graph $W$ contains a distinguished vertex $w$. There are $n=|\mathcal{V}(Q)|$ edges incident on $w$. Let $e$ be such an edge; one endpoint of $e$ equals $w$. Attached to the second endpoint is a circle with $k^{2^{h-1}}$ vertices.

Note that $W$ admits a group of automorphisms which fix $w$ and permute the edges of $W$ incident on $w$. Each permutation of the edges incident on $w$ is the restriction of such an automorphism. Any labeling of the edges of $W$ incident on $w$ gives rise to a bijection $\mathcal{V}(H) \rightarrow \mathcal{V}(W)-\{w\}$ which maps 
the vertices in $Q$ to endpoints of the edges incident on $w$. Fix once and for all such a bijection $\Theta$.

Via the map $\Theta$, each function $f \in E$ naturally induces a function $f^{*}$ on $\mathcal{V}(W)$. This function may not be of zero mean, but as $f$ is of zero mean and the map $\Theta$ is a bijection of set of the vertices of $H$ onto the set of vertices of $W$ distinct from $w$, the square norm of the normalization $g$ of $f^{*}$ is not smaller than $\sum_{v \in H} f^{2}(v)$ (compare the proof of Proposition 3.2).

As $f \in E$, as $\Theta$ maps vertices of degree contained in $[2,4 h]$ to vertices of degree in $[2,3]$, and as the special vertex $w$ does not contribute to $\int\left(g^{\prime}\right)^{2}$, we have $\int\left(g^{\prime}\right)^{2} \leq 4 h \int\left(f^{\prime}\right)^{2}$. Together this shows

$$
\mathcal{R}(f) \geq \frac{1}{4 h} \mathcal{R}(g) .
$$

As a consequence, for the desired estimate of $\lambda_{1}(E)$ it suffices to show that $\lambda_{1}(W) \geq m / k^{2^{h}}$ for a universal constant $m>0$.

To this end let $f$ be an eigenfunction on $W$ for the smallest eigenvalue $\lambda_{1}(W)$. If we define

$$
\mathcal{L} f(u)=\frac{1}{\sqrt{p(v)}} \sum_{w \sim v}\left(\frac{f(v)}{\sqrt{p(v)}}-\frac{f(w)}{\sqrt{p(w)}}\right)
$$

then $\mathcal{L} f(u)=\lambda_{1}(W) f(u)$.

We distinguish now two cases. In the first case, $f(w)=0$. Then equation (12) shows that the restriction $f_{U}$ of $f$ to the closure of each component $U$ of $W-\{w\}$ is an eigenfunction on $U$ for the eigenvalue $\lambda_{1}(W)$. Such a component $U$ is a circle of length $k^{2^{h-1}}$ with a single edge attached at one vertex. As $f_{U}$ assumes the value zero, its Rayleigh quotient $\mathcal{R}(f \mid U)$ can be estimated by

$$
\mathcal{R}(f \mid U) \succeq\left(k^{2^{h-1}}\right)^{2}=k^{2^{h}}
$$

by the discussion in the beginning of this proof which is equally valid for a circle with a single edge attached at one vertex instead of a circle.

Together this yields

$$
\begin{array}{r}
\int\left(f^{\prime}\right)^{2} \geq \sum_{U} \sum_{v \in \mathcal{V}(U)-\{w\}} \frac{1}{p(v)} \sum_{z \sim v}(f(z)-f(v))^{2} \\
\succeq\left(\sum_{U} \sum_{v \in \mathcal{V}(U)-\{w\}} f^{2}(v)\right) / k^{2^{h}}
\end{array}
$$

and therefore $\mathcal{R}(f) 0 \lambda_{1}(W) \succeq 1 / k^{2^{h}}$ as desired.

Now assume that $f(w) \neq 0$. Let $A$ be an automorphism of $W$; then $f \circ A$ is an eigenfunction for the eigenvalue $\lambda_{1}(W)$. If $f \circ A \neq f$, then $f-f \circ A$ is an eigenfunction on $W$ for the eigenvalue $\lambda_{1}(W)$ which vanishes at $w$. The desired estimate now follows from the above discussion provided that $f \circ A \neq A$ for at least one automorphism $A$ of $W$.

Finally suppose that $f \circ A=f$ for all automorphisms $A$ of $W$. Then $f$ descends to an eigenfunction $\hat{f}$ on a circle $U$ of length $k^{2^{h-1}}$ with a single 
edge attached at one vertex, and of zero mean. Here the value of $\hat{f}$ at the unique vertex of $U$ of degree one equals $f(w) /|\mathcal{V}(Q)|$. The eigenvalue of $\hat{f}$ equals $\lambda_{1}(W)$. Then $\lambda_{1}(W) \geq \lambda_{1}(U)$ and hence as before, we conclude that $\lambda_{1}(W) \succeq 1 / k^{2^{h}}$ for a universal constant $q$. Together this shows that indeed $\lambda_{1}(E) \succeq 1 / k^{2^{h}}$ as claimed.

Since we have established that $\lambda_{1}(D) \succeq 1 / k^{2^{h}}$ and $\lambda_{1}(E) \succeq 1 / k^{2^{h}}$, we get $\lambda_{1}(G) \asymp \lambda_{1}(H) \succeq 1 / k^{2^{h}}$. This shows the proposition.

Remark 3.5. The constant $q(h)$ in Proposition 3.4 can be made effective. However, this would require a considerable effort in bookkeeping. Moreover, our proof would yield an exponential decay of $q(h)$ in $h$.

\section{The SMAllest eigenvalue of MAPPInG TORI}

In this section we use the results from Section 3 and Section 2 to prove Theorem 1 from the introduction.

As explained in Section 2, a hyperbolic 3-manifold $M$ can be decomposed as $M=M_{\text {thick }} \cup M_{\text {thin }}$ where $M_{\text {thick }}, M_{\text {thin }}$ are smooth manifolds with boundary $\partial M_{\text {thick }}, \partial M_{\text {thin }}$. Each component of $M_{\text {thin }}$ is a Margulis tube. Such a tube $T$ is a tubular neighborhood of a geodesic $\gamma$ in $M$ of length less than $2 \varepsilon$ where $\varepsilon>0$ is a Margulis constant for hyperbolic 3-manifolds. The geodesic $\gamma$ is called the core geodesic of the tube.

The thick part $M_{\text {thick }}$ of $M$ is a smooth submanifold of $M$ with boundary. Thus the spectrum of $M_{\text {thick }}$ with Neumann boundary conditions is defined. This spectrum is discrete, with finite multiplicities. Constant functions are eigenfunctions corresponding to the smallest eigenvalue $\lambda_{0}=0$. Let $\lambda_{1}\left(M_{\text {thick }}\right)$ be the smallest non-zero eigenvalue with Neumann boundary conditions. We now evoke the main result of [22] to show

Proposition 4.1. For every $g \geq 2$ there is a number $c_{3}=c_{3}(g)>0$ with the following property. Let $M$ be a hyperbolic mapping torus of genus $g$; then

$$
\lambda_{1}\left(M_{\text {thick }}\right) \leq c_{3} / \operatorname{vol}(M)^{2^{2 g-2} /\left(2^{2 g-2}-1\right)} .
$$

Proof. By Proposition 2.2, $M_{\text {thick }}$ is $L$-quasi-isometric to a generalized array $G$ of circles of depth at most $2 g-2$ for a number $L=L(g)>0$ only depending on $g$. The main result of [22] applies to the Laplacian on manifolds with boundary and Neumann boundary condition (see [19] for a more precise statement along these lines) and shows that there is a number $b>0$ only depending on $g$ so that $\lambda_{1}\left(M_{\text {thick }}\right) \leq b \lambda_{1}(G)$.

Proposition 3.2 yields that

$$
\lambda_{1}(G) \leq 256 \pi^{2}(2 g-2)^{2 g-3} / \operatorname{vol}(G)^{2^{2 g-2} /\left(2^{2 g-2}-1\right)} .
$$

The proposition now follows from the fact that $\operatorname{vol}(G) \sim \operatorname{vol}\left(M_{\text {thick }}\right)$ (by uniform quasi-isometry) and $\operatorname{vol}\left(M_{\text {thick }}\right) \geq \frac{2}{3} \operatorname{vol}(M)$ for a suitable choice of a Margulis constant (by the explicit description of the metric in a Margulis tube, see [13] for a more comprehensive discussion). 
The following is shown in [13].

Proposition 4.2. There exists a number $d>0$ and a suitable choice of a Margulis constant such that

$$
\frac{1}{48} \lambda_{1}\left(M_{\text {thick }}\right) \leq \lambda_{1}(M) \leq d \log \operatorname{vol}\left(M_{\text {thin }}\right) \lambda_{1}\left(M_{\text {thick }}\right)
$$

for every hyperbolic 3-manifold $M$.

We are now ready to show Theorem 1 from the introduction.

Corollary 4.3. For every $g \geq 2$ there is a number $C_{1}=C_{1}(g)>0$ with the following properties.

$$
\lambda_{1}(M) \leq C_{1} \log \operatorname{vol}(M) / \operatorname{vol}(M)^{2^{2 g-2} /\left(2^{2 g-2}-1\right)} .
$$

for every hyperbolic mapping torus $M$ of genus $g$.

(2) There exists a sequence $M_{i}$ of hyperbolic mapping tori of genus $g$ with $\operatorname{vol}\left(M_{i}\right) \rightarrow \infty$ and such that

$$
\lambda_{1}\left(M_{i}\right) \geq C_{1}^{-1} / \operatorname{vol}\left(M_{i}\right)^{2^{2 g-2} /\left(2^{2 g-2}-1\right)} .
$$

Proof. The first part of the corollary is immediate from Proposition 4.1 and from Proposition 4.2 .

To show the second part, let $g \geq 2$ be arbitrary. By Proposition 2.5 , there exists a sequence $M_{i}$ of mapping tori of genus $g$ so that $\operatorname{vol}\left(M_{i}\right) \rightarrow \infty$ and that for each $i,\left(M_{i}\right)_{\text {thick }}$ is uniformly quasi-isometric to an optimal step homogeneous array of circles $G_{i}$ of depth $2 g-2$.

By Proposition 3.4, the first eigenvalue of the array $G_{i}$ is not smaller than $\hat{q} / \operatorname{vol}\left(G_{i}\right)^{2^{2 g-2} / 2^{2 g-2}-1}$ where $\hat{q}=\hat{q}(2 g-2)$ is a universal constant.

Using once more the main result of [22] and the volume comparison

$$
\operatorname{vol}\left(G_{i}\right) \asymp \operatorname{vol}\left(\left(M_{i}\right)_{\text {thick }}\right) \geq \frac{2}{3} \operatorname{vol}\left(M_{i}\right)
$$

for a suitable choice of a Margulis constant, we conclude that there is a universal constant $c^{\prime}>0$ so that

$$
\lambda_{1}\left(\left(M_{i}\right)_{\text {thick }}\right) \geq c^{\prime} / \operatorname{vol}\left(M_{i}\right)^{2^{2 g-2} /\left(2^{2 g-2}-1\right)} .
$$

The second part of the corollary now follows from the first inequality of Proposition 4.2 .

We complete this section by estimating the smallest eigenvalue of mapping tori defined by periodic Teichmüller geodesics in moduli space $\mathcal{M}(S)$ which spend a definite proportion of time in the $\varepsilon$-thick part $\mathcal{M}(S)_{\varepsilon}$ of surfaces with injectivity radius bigger than $\varepsilon$. Note that $\mathcal{M}(S)_{\varepsilon}$ is the quotient of the $\varepsilon$-thick part of Teichmüller space under the action of the mapping class group. 
Proposition 4.4. For sufficiently small $\varepsilon>0$ there exists a number $b=$ $b(g, \varepsilon)>0$ with the following property. Let $S_{R}^{1}$ be the circle of length $R>0$ and let $\gamma: S_{R}^{1} \rightarrow \mathcal{M}(S)$ be a periodic Teichmüller geodesic of length $R$. Let $p=3 c_{2}(g, \varepsilon)>0$ be as in Proposition [2.6 and let $Q=\left\{t \in S_{R}^{1}\right.$ | $\left.\gamma(t-p, t+p) \subset \mathcal{M}(S)_{\varepsilon}\right\}$; if the Lebesgue measure of $Q$ is at least $\zeta R$ for some $\zeta \in(0,1)$ then

$$
\lambda_{1}(M) \leq \frac{b}{\zeta^{2} \operatorname{vol}(M)^{2}} .
$$

Proof. Let $c_{2}=c_{2}(g, \varepsilon)>0$ be as in Proposition 2.6. let $p=3 c_{2}$ and let $\zeta \in(0,1)$. Let $\gamma: S_{R}^{1} \rightarrow \mathcal{M}(S)$ be a periodic Teichmüller geodesic as in the proposition for this number $\zeta$. By continuity, the set $Q \subset S_{R}^{1}$ is open and hence it is a union of at most countably many open intervals. By the definition of $Q$, the $p$-neighborhoods of these intervals are pairwise disjoint. By assumption, the Lebesgue measure of $Q$ is at least $\zeta R$. Choose finitely many connected components $I_{1}, \ldots, I_{s} \subset Q$ of Lebesgue measure at least $\zeta R / 2$. Assume that these intervals are linearly ordered along $[0, R]$. Let $u_{j}$ be the length of $I_{j}$.

By Proposition 2.6, for each $j \leq s$ there exists a submanifold $N_{j}$ of $M$ with smooth boundary which is diffeomorphic to $S \times[0,1]$. This diffeomorphism is chosen to be compatible with the orientation of $S_{R}^{1}$ defined by the parametrization of $\gamma$. Thus the two boundary components of $N_{j}$ are naturally ordered. We denote by $\partial N_{j}^{-}$the component which is smaller for this order, and by $\partial N_{j}^{+}$the bigger component. The submanifolds $N_{j}$ are pairwise disjoint, and $M-\cup_{i} N_{i}$ consists of $s$ connected components $P_{1}, \ldots, P_{s}$ diffeomorphic to $S \times[0,1]$. We have $\partial P_{i}=\partial N_{i}^{+} \cup \partial N_{i+1}^{-}$.

For each $j$ there exists a smooth surjective map

$$
f_{j}: N_{j} \rightarrow\left[\sum_{i<j} u_{i}, \sum_{i \leq j} u_{j}\right]
$$

of uniformly bounded derivative. Write $u=\sum_{i} u_{i} \geq \zeta R / 2$ and define a function $f: M \rightarrow[0, u]$ by $f \mid N_{i}=f_{i}$ and by the requirement that $f$ is constant on each of the manifolds $P_{j}$. We can modify $f$ so that its derivative is uniformly bounded. Define functions $\alpha, \beta$ on $[0, u]$ as

$$
\alpha(s)= \begin{cases}\sin (\pi s / u), & \text { if } 0 \leq s \leq u / 2 \\ 0 & \text { if } u / 2 \leq s \leq u\end{cases}
$$

and

$$
\beta(s)= \begin{cases}0, & \text { if } 0 \leq s \leq u / 2 \\ \sin (\pi(s-u / 2) / u) & \text { if } u / 2 \leq s \leq u\end{cases}
$$

Then $\alpha \circ f, \beta \circ f$ are smooth, with supports intersecting in a zero volume set, and their Rayleigh quotients are uniformly equivalent to $1 / u^{2}$. To this end note that the Rayleigh quotients of $\alpha, \beta$ are $\pi^{2} / u^{2}$, and since $f$ has uniformly bounded derivative, the Rayleigh quotients of $\alpha, \beta$ are uniformly equivalent to the Rayleigh quotients of $\alpha \circ f, \beta \circ f$. 
By the Minmax-theorem for the spectrum of the Laplacian, we know that for any set of functions $\rho_{0}, \ldots, \rho_{k}: M \rightarrow \mathbb{R}$ whose supports pairwise intersect on zero-volume sets, we have $\lambda_{k} \leq \max \left\{\mathcal{R}\left(\rho_{i}\right) \mid 0 \leq i \leq k\right\}$ (compare [34]) and therefore $\lambda_{1}(M) \leq \max \{\mathcal{R}(\alpha \circ f), \mathcal{R}(\beta \circ f)\}$. As a result, $\lambda_{1}(M) \leq d / u^{2}$ where $d>0$ is a constant only depending on $g, \varepsilon$.

We are left with showing that for fixed $\zeta$, the volume of $M$ is uniformly equivalent to $u$. To this end we evoke from [2, 3, 16] that the volume of $M$ is equivalent to the translation length for the Weil-Petersson metric of the pseudo-Anosov element defining $\gamma$, and this translation length is bounded from above by the length of $\gamma$ for the Teichmüller metric up to a factor which only depends on $g$ (see e.g. [16]). Thus the volume of $M$ is bounded from above by $\chi R$ where $\chi>0$ only depends on $\zeta, g, \varepsilon$ and is linear in $\zeta$. Since $u \geq \zeta R / 2$, the proposition follows.

\section{TYPICAL MAPPING TORI}

The goal of this section is to show that a typical mapping torus satisfies the hypothesis in Proposition 4.4 for a number $\zeta>0$ only depending on the genus for some fixed sufficiently small number $\varepsilon>0$. We then evoke Proposition 4.4 to conclude the proof of Theorem 2 for typical mapping tori.

Let $\mathcal{G}(L)$ be the set of all conjugacy classes of pseudo-Anosov mapping classes (in short: p-A mapping classes) on $S$ whose translation length on Teichmüller space $\mathcal{T}(S)$ is less than $L$. It is known that $\mathcal{G}(L)$ is finite for any fixed $L>0$. Up to isometry, a hyperbolic mapping torus only depends on the conjugacy class of the defining pseudo-Anosov element. We say a typical mapping torus (or a typical p-A conjugacy class) satisfies a property $(*)$ if

$$
\frac{\mid\{\phi \in \mathcal{G}(L): \phi \text { satisfies property }(*)\} \mid}{|\mathcal{G}(L)|} \rightarrow 1
$$

as $L \rightarrow \infty$. In this section we prove the following.

Proposition 5.1. Let $U \subset \mathcal{T}(S)$ be an open $\operatorname{Mod}(S)$-invariant set which contains the axis of at least one pseudo-Anosov element. For each $p>0$, there exists $\delta=\delta(U, p)>0$ with the following property. The proportion of time along an axis $\gamma$ of a typical pseudo-Anosov element consisting of points $\gamma(t)$ so that the segment $\gamma[t-p, t+p]$ is entirely contained in $U$ is at least $\delta$.

To prove this proposition, we will use the equidistribution of closed orbits of the Teichmüller geodesic flow in the space of unit area quadratic differentials, obtained in [11. Let $\widetilde{Q^{1}(S)} \rightarrow \mathcal{T}(S)$ be the bundle of unit area quadratic differentials, which can be identified with the unit cotangent bundle to $\mathcal{T}(S)$, and let $Q^{1}(S)=\widetilde{Q^{1}(S)} / \operatorname{Mod}(S)$.

The Teichmüller flow $\Phi^{t}: Q^{1}(S) \rightarrow Q^{1}(S)$ acts on $Q^{1}(S)$ preserving a finite measure $\lambda$ in the Lebesgue measure class, the Masur-Veech measure. The measure $\lambda$ has full support. 
We can identify conjugacy classes of pseudo-Anosov elements with closed orbits of the Teichmüller geodesic flow.

For a closed orbit $\gamma$ let $l(\gamma)$ denote its length.

Let $\delta_{\gamma}$ be the standard flow-invariant Lebesgue measure on $\gamma$ of total mass $l(\gamma)$.

For a Borel subset $A$ of $\mathcal{Q}^{1}(S)$ let $l(A)=\delta_{\gamma}(A)$.

For each $L>0$ we may define a measure on $\mathcal{Q}^{1}(S)$ by

$$
\lambda_{L}=\frac{1}{L|\mathcal{G}(L)|} \sum_{\gamma \in \mathcal{G}(L)} \delta_{\gamma} .
$$

The main result of [1] shows:

Lemma 5.2. The measures

$$
\lambda_{L}=\frac{1}{L|\mathcal{G}(L)|} \sum_{\gamma \in \mathcal{G}(L)} \delta_{\gamma}
$$

weakly converge to $\lambda$ as $L \rightarrow \infty$.

By the classical Portmanteau theorem Lemma 5.2 can be rephrased as follows.

Lemma 5.3. For any Borel set $V \subset Q^{1}(S)$ whose boundary has measure zero we have

$$
\lim _{L \rightarrow \infty} \frac{1}{L|\mathcal{G}(L)|} \sum_{\gamma \in \mathcal{G}(L)} l(\gamma \cap V)=\lambda(V)
$$

We will use Lemma 5.2 together with the ergodicity of the Teichmüller geodesic flow to prove the following.

Proposition 5.4. Let $U \subset Q^{1}(S)$ be a Borel subset whose boundary has Lebesgue measure zero. Then for any $\varepsilon>0$ a typical Teichmüller geodesic spends a proportion between $(1-\varepsilon) \mu(U)$ and $(1+\varepsilon) \mu(U)$ in $U$.

Proof. It suffices to prove that for each $\varepsilon>0$, a typical closed orbit spends a proportion at least $(1-\varepsilon) \mu(U)$ in $U$ (the upper bound can then be obtained by replacing $U$ with its complement). Fix $\varepsilon>0$. For each $L>0$ let $A(L) \subset \mathcal{G}(L)$ denote the set corresponding to closed orbits of length at most $L$ that spend a proportion at most $(1-\varepsilon) \lambda(U)$ in $U$. Define for each $L>0$ a finite measure

$$
\kappa_{L}=\frac{1}{L|\mathcal{G}(L)|} \sum_{\gamma \in \mathcal{G}(L)} \delta_{\gamma} .
$$

To prove Proposition 5.4 it suffices to prove that the measures $\kappa_{L}$ weakly converge to zero. Note, $\kappa_{L} \leq \lambda_{L}$ so by Lemma 5.2 any subsequence of the $\kappa_{L}$ has an weak accumulation point, which is a finite measure on $Q^{1}(S)$.

Let $\kappa$ be the weak limit of $\kappa_{L_{i}}$ for some sequence $L_{i}$. As $\kappa_{L}(U) \leq(1-$ $\varepsilon) \kappa_{L}\left(Q^{1}(S)\right)$ for each $L$ and the boundary of $U$ has measure 0 we have

$$
\kappa(U) \leq(1-\varepsilon) \kappa\left(Q^{1}(S)\right) .
$$


Since the $\kappa_{L}$ are $\Phi^{t}$ invariant so is $\kappa$. Thus, by ergodicity of the Teichmüller flow with respect to $\lambda$ we have $\kappa=c \lambda$ for some $c=\kappa\left(Q^{1}(S)\right) \geq 0$. Then $\kappa(U)=\lambda(U) \kappa\left(Q^{1}(S)\right)$, providing a contradiction and completing the proof.

We now conclude the proof of Proposition 5.1,

Proof of Proposition 5.1. Let $U \subset \mathcal{T}(S)$ be a nonempty open set containing an axis of a pseudo-Anosov element. Let $W$ be the preimage of $U$ in $\widetilde{Q^{1}(S)}$ and $V$ the image of $W$ in $Q^{1}(S)$. For $T>0$, let $V_{T} \subset Q^{1}(S)$ be the subset of $q$ such that $\Phi^{t} q \in V$ for all $t \in(-T, T)$. By finiteness of $\lambda$ we know that $\lambda\left(\partial V_{T}\right)=0$ for all but countable many $T$, so for given $p>0$ we may find a $T>p$ with this property. Then by Proposition 5.4 for any $\varepsilon>0$ a typical closed orbit of the Teichmüller flow spends a proportion at least $(1-\varepsilon) \mu\left(V_{T}\right)$ in $V$. Thus, the proportion of time along an axis $\gamma$ of a typical pseudoAnosov element consisting of points $\gamma(t)$ so that the segment $\gamma[t-p, t+p]$ is entirely contained in $U$ is at least $\mu\left(V_{T}\right)$. Since $U$ is open so is $V_{T}$, and since $U$ contains an axis of a pseudo-Anosov element we know $U_{T}$ is nonempty so $\mu\left(V_{T}\right)>0$. This completes the proof with $\delta(p)=\lambda\left(V_{T}\right) / 2>0$.

As a result, we obtain Theorem 2 for typical mapping tori.

Corollary 5.5. For every $g \geq 2$ there exists a number $\kappa=\kappa(g)>0$ so that

$$
\lambda_{1}(M) \in\left[\frac{1}{\kappa(g) \operatorname{vol}(M)^{2}}, \frac{\kappa(g)}{\operatorname{vol}(M)^{2}}\right]
$$

for a typical mapping torus of genus $g$.

Proof. Choose $\varepsilon>0$ sufficiently small that the open $\operatorname{Mod}(S)$-invariant subset $\mathcal{T}(S)_{\varepsilon} \subset \mathcal{T}(S)$ of all surfaces whose systole is bigger $\varepsilon$ contains the axis of a pseudo-Anosov element. Let $p=3 c_{2}(g, \varepsilon)>0$ as in Proposition 2.6. Proposition 5.1 shows there exists a number $\zeta>0$ such that for a typical periodic Teichmüller geodesic $\gamma$, the proportion of time $t$ so that the segment $\gamma(t-p, t+p)$ is entirely contained in $\mathcal{M}(S)_{\varepsilon}$ is at least $\zeta$. The corollary now follows from Proposition 4.4.

\section{RANDOM MAPPING TORI}

The main goal of this section is to prove Theorem 3 from the introduction. The part of Theorem 2 concerning radom mapping tori follows from this and Proposition 4.4.

We begin with reviewing some background on random walks on groups. This is a vast subject, see for example [15] [7] [21] for more details.

Let $G$ be a countable finitely generated group. Let $\mu$ be a symmetric probability measure on $G$ and let $\mu^{\mathbb{Z}}$ be the product measure on $G^{\mathbb{Z}}$.

Let $T: G^{\mathbb{Z}} \rightarrow G^{\mathbb{Z}}$ be the following invertible transformation: $T$ takes the two-sided sequence $\left(h_{i}\right)_{i \in \mathbb{Z}}$ to the sequence $\left(g_{i}\right)_{i \in \mathbb{Z}}$ with $g_{0}=e$ and 
$g_{n}=g_{n-1} h_{n}$ for $n \neq 0$. Explicitly, this means

$$
g_{n}=h_{1} \cdots h_{n} \quad \text { for } n>0
$$

and

$$
g_{n}=h_{0}^{-1} h_{-1}^{-1} \cdots h_{-n+1}^{-1} \quad \text { for } n<0 .
$$

Similarly, let $\mu^{\mathbb{N}}$ be the product measure on $G^{\mathbb{N}}$. Let $T_{+}: G^{\mathbb{N}} \rightarrow G^{\mathbb{N}}$ be the transformation that takes the one-sided infinite sequence $\left(h_{i}\right)_{i \in \mathbb{N}}$ to the sequence $\left(g_{i}\right)_{i \in \mathbb{N}}$ with $g_{0}=e$ and $g_{n}=g_{n-1} h_{n}$ for $n \neq 0$. Explicitly, for $n>0$ this means

$$
g_{n}=h_{1} \cdots h_{n}
$$

Let $\bar{P}$ be the pushforward measure $T^{*} \mu^{\mathbb{Z}}$ and $P$ the pushforward measure $T_{+}^{*} \mu^{\mathbb{N}}$.

The measure $P$ describes the distribution $\mu$ on sample paths, i.e. of products of independent $\mu$-distributed increments. The measure space $\left(G^{\mathbb{Z}}, \bar{P}\right)$ is naturally isomorphic to $\left(G^{\mathbb{N}}, P\right) \otimes\left(G^{\mathbb{N}}, P\right)$ via the map sending the bilateral path $\omega$ to the pair of unilateral paths $\left(\left(\omega_{n}\right)_{n \in \mathbb{N}},\left(\omega_{-n}\right)_{n \in \mathbb{N}}\right)$.

Assume now that $G$ acts by isometries on a metric space $\left(X, d_{X}\right)$ and let $x_{0} \in X$. If $\mu$ has finite first moment (which is obviously the case if the support of $\mu$ is finite, which is the case of interest for us), Kingman's subadditive ergodic theorem implies that for $P$ a.e. sample path $\omega$ the limit

$$
L_{X}=\lim _{n \rightarrow \infty} \frac{d_{X}\left(\omega_{n} x_{0}, x_{0}\right)}{n}
$$

exists. This number $L$ is called the drift of the random walk induced by $\mu$ with respect to the metric $d_{X}$.

In the case that $\left(X, d_{X}\right)$ is a separable Gromov hyperbolic metric space then the action of $G$ on $X$ is called nonelementary if $G$ contains a pair of loxodromic isometries with disjoint sets of fixed points in the Gromov boundary $\partial X$ of $X$. A symmetric probability measure on $G$ is called nonelementary if the subgroup of $\mathrm{G}$ generated by its support is a nonelementary subgroup of $G$. The following results are due in this generality to Maher and Tiozzo 21].

Theorem 6.1. Let $G$ be a countable group that acts by isometries on a separable Gromov hyperbolic space $\left(X, d_{X}\right)$ such that any two points in $X \cup$ $\partial X$ can be connected by a geodesic. Let $\mu$ be a nonelementary probability measure on $G$.

(1) For any $x \in X$ and $P$ a.e. sample path $\omega=\left(\omega_{n}\right)_{n \in \mathbb{N}}$ of the random walk on $(G, \mu)$, the sequence $\left(\omega_{n} x_{0}\right)_{n \in \mathbb{N}}$ converges to a point $\operatorname{bnd}(\omega) \in \partial X$.

(2) If $\mu$ has finite first moment with respect to the metric $d_{X}$, then there exists $L_{X}>0$ such that for $P$-a.e. sample path $\omega$ one has

$$
\lim _{n \rightarrow \infty} \frac{d_{X}\left(x_{0}, \omega_{n} x_{0}\right)}{n}=L_{X}
$$


Moreover, there is a unit speed geodesic ray $\tau$ converging to bnd $(\omega)$ such that

$$
\lim _{n \rightarrow \infty} \frac{d_{X}\left(\tau\left(L_{X} n\right), \omega_{n} x_{0}\right)}{n}=0 .
$$

(3) If $\mu$ has finite support, then for P-a.e. sample path $\omega$ there is an $n_{0}$ such that $\omega_{n}$ acts loxodromically for all $n \geq n_{0}$.

Using techniques of [21], Dahmani and Horbez (Proposition 1.9 of [7]) proved.

Proposition 6.2. Let $X$ be a separable geodesic Gromov hyperbolic metric space, with hyperbolicity constant $\delta$. For all $A>0$, there exists a number $\kappa=\kappa(A, \delta)>0$ such that the following holds. Let $G$ be a group acting by isometries on $X$, and let $\mu$ be a nonelementary probability measure on $G$ with finite support. Let $L_{X}>0$ denote the drift of the random walk on $(G, \mu)$ with respect to $d_{X}$. Then for $P$-a.e. sample path $\omega$ of the random walk on $(G, \mu)$, for all $\varepsilon>0$, and all $A$-quasi-geodesic rays $\gamma: R^{+} \rightarrow X$ converging to $\operatorname{bnd}(\omega) \in \partial X$, there exists $n_{0} \in \mathbb{N}$ such that for all $n \geq n_{0}$, any $A$-quasiaxis of $\omega_{n}$ intersects the $\kappa$-neighborhood of $\gamma\left[t_{1}(n), t_{2}(n)\right]$. Here $t_{1}(n)$ (resp. $\left.t_{2}(n)\right)$ is the infimum of all real numbers such that $d_{X}\left(\gamma(0), \gamma\left(t_{1}(n)\right)\right) \geq$ $\varepsilon L_{X} n\left(\operatorname{resp} . d_{X}\left(\gamma(0), \gamma\left(t_{2}(n)\right)\right) \geq(1-\varepsilon) L_{X} n\right)$.

We will apply these results to $G=\operatorname{Mod}(S)$, the mapping class group of the surface $S$. It acts on the associated complex of curves $X=\mathcal{C}(S)$, which is Gromov hyperbolic by work of Masur and Minsky [24], and has the property that any two points in $X \cup \partial X$ can be connected by a geodesic by work of Bowditch [1]. An element of $\operatorname{Mod}(S)$ acts as a loxodromic isometry on $\mathcal{C}(S)$ if and only if it is a pseudo-Anosov. We will call a subgroup of $\operatorname{Mod}(S)$ or a measure on $\operatorname{Mod}(S)$ nonelementary if it is nonelementary for the action on $\mathcal{C}(S)$.

Our main technical result (Proposition 6.11) is an analog of Proposition 6.2 for the action of $\operatorname{Mod}(S)$ on the Teichmüller space $\mathcal{T}(S)$ with the Teichmüller metric $d_{T}$ and its Thurston boundary $\mathcal{P} \mathcal{M L}$ of all measured projective laminations. We begin with collecting those known results for this action we shall use for our goal.

The set $\mathcal{P} \mathcal{M L}$ contains the invariant subset of uniquely ergodic laminations. In the following results of Kaimanovich and Masur [15], The notion of a non-elementary probability measure is the notion discussed above.

Theorem 6.3. Let $\mu$ be a nonelementary probability measure on $\operatorname{Mod}(S)$. For $P$-almost every $\omega$, and every o $\in \mathcal{T}(S), \omega_{n}$ o converges to a uniquely ergodic point $\operatorname{bnd}_{T}(\omega) \in \mathcal{P} \mathcal{M L}$.

In other words, there is a $P$-almost everywhere defined measurable map bnd : $G^{\mathbb{N}} \rightarrow \mathcal{P} \mathcal{M L}$ sending $\omega$ to $\lim _{n \rightarrow \infty} \omega_{n} o \in \mathcal{P} \mathcal{M L}$. The measure on $\mathcal{P} \mathcal{M L}$ defined by

$$
\nu=\operatorname{bnd}_{*} P=\lim _{n \rightarrow \infty} \mu^{* n}
$$


is called the harmonic measure for $\mu$. In fact, $(\mathcal{P} \mathcal{M L}, \nu)$ is a model for the Poisson boundary of $(G, \mu)$ [15]. In particular, $\nu$ is the unique $\mu$ stationary measure on $\mathcal{P} \mathcal{M L}$ : for every $g \in G$ and $\nu$ measurable $V \subset \mathcal{P} \mathcal{M L}$ we have

$$
\nu(V)=\sum_{g \in G} \mu(g) \nu\left(g^{-1} V\right)
$$

and consequently for every $n>0$ :

$$
\nu(V)=\sum_{g \in G} \mu^{* n}(g) \nu\left(g^{-1} V\right) .
$$

The stationarity and the fact that the support of $\mu$ generates $G$ implies that if $\nu(V)>0$ then $\nu(g V)>0$ for every $g \in G$. For every $g \in G$ we have:

$$
P\left(\omega: \lim _{n \rightarrow \infty} g \omega_{n} \in V\right)=P\left(\omega: \lim _{n \rightarrow \infty} \omega_{n} \in g^{-1} V\right)=\nu\left(g^{-1} V\right)
$$

If $C_{e, h_{1}, \ldots h_{k}}$ is the cylinder subset of $G^{\mathbb{N}}$ consisting of $\omega$ with $\omega_{i}=h_{i}$ for $i \leq k$ we have

$$
\begin{gathered}
P\left(C_{e, h_{1}, \ldots h_{k}} \cap \mathrm{bnd}^{-1} V\right) \\
=P\left(C_{e, h_{1}, \ldots h_{k}}\right) P\left(\omega: \lim _{n \rightarrow \infty} h_{k} \omega_{n} \in V\right)=P\left(C_{e, h_{1}, \ldots h_{k}}\right) \nu\left(h_{k}^{-1} V\right)
\end{gathered}
$$

(this is proved in general for the Poisson boundary by Kaimanovich in Section 3.2 of [14]). In particular, if $\nu(V)>0$ then for any cylinder subset $C \subset G^{\mathbb{N}}$ we have $P\left(C \cap\right.$ bnd $\left.^{-1} V\right)>0$.

The following claim follows from minimality of the action of $G$ on $\mathcal{P} \mathcal{M L}$.

Lemma 6.4. The measure $\nu$ has full support on $\mathcal{P} \mathcal{M L}$.

Proof. Let $V \subset \mathcal{P} \mathcal{M L}$ be an open set. By minimality, $\bigcup_{g \in G} g V=\mathcal{P} \mathcal{M L}$, and hence $\nu\left(\bigcup_{g \in G} g V\right)=1>0$ whence $\nu(g V)>0$ for some $g \in G$. By stationarity, $\nu(V)>0$.

The analog of Theorem 6.1 for the action of the mapping class group on $\mathcal{T}(S)$ is due to Tiozzo [33].

Theorem 6.5. Let $\mu$ be a nonelementary probability measure on $\operatorname{Mod}(S)$ with finite first moment for $d_{T}$. Then there exists $L_{T}>0$ such that for $P$-a.e. sample path $\omega$ one has

$$
\lim _{n \rightarrow \infty} \frac{d_{T}\left(o, \omega_{n} o\right)}{n}=L_{T}
$$

and for any geodesic ray $\tau$ converging to $\operatorname{bnd}_{T}(\omega)$ we have

$$
\lim _{n \rightarrow \infty} \frac{d_{T}\left(\tau\left(L_{T} n\right), \omega_{n} o\right)}{n}=0 .
$$

For a pseudo-Anosov element $\phi \in \operatorname{Mod}(S)$ let $l(\phi)$ be its translation length in $\mathcal{T}(S)$. The following result is Theorem 3.1 of [7].

Theorem 6.6. Let $\mu$ be a nonelementary probability measure on $\operatorname{Mod}(S)$ with finite support. Then $l\left(\omega_{n}\right) / n \rightarrow L_{T}(n \rightarrow \infty)$ for $P$ almost every $\omega$. 
Reformulating the above discussion in terms of bilateral sample paths instead we obtain:

Theorem 6.7. For $\mu$-almost every sample path $\omega \in G^{\mathbb{Z}}$ and every $x \in \mathcal{T}(S)$ the limits

$$
\operatorname{bnd}_{ \pm}(\omega)=\lim _{n \rightarrow \pm \infty} \omega_{n} x \in \mathcal{P} \mathcal{M L}
$$

exist, are independent of $x$, distinct and uniquely ergodic.

There is a geodesic $\tau_{\omega}$ with vertical and horizontal foliations $\operatorname{bnd}^{ \pm}(\omega)$, and for any unit speed parametrization of $\tau_{\omega}$ we have

$$
\lim _{n \rightarrow \pm \infty} d_{T}\left(x, \omega_{n} x\right) /|n| \rightarrow L_{T}
$$

and

$$
\lim _{n \rightarrow \pm \infty} d_{T}\left(\tau_{\omega}\left(L_{T} n\right), \omega_{n} x\right) /|n| \rightarrow 0 .
$$

The measure $\nu=\operatorname{bnd}_{*} P$ has full support on $\mathcal{P} \mathcal{M L}$. Moreover, for any set $V \subset \mathcal{P} \mathcal{M L} \times \mathcal{P} \mathcal{M L}$ with $(\nu \otimes \nu)(V)>0$ and each cylinder subset $B$ of $G^{\mathbb{Z}}$ we have $\bar{P}\left(\left(\text { bnd }_{-} \times \text {bnd }_{+}\right)^{-1}(V) \cap B\right)>0$.

From now on, for $o \in \mathcal{T}(S)$ and for almost every sample path $\omega \in$

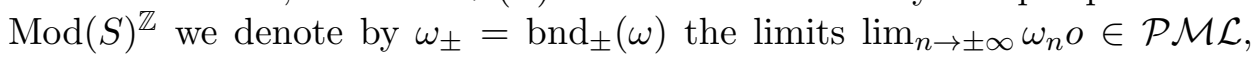
respectively. Moreover, $\tau_{o, \omega_{+}}$denotes the Teichmüller geodesic ray which connects the basepoint $o$ to $\omega_{+}$. Recall that this makes sense since for $\bar{P}$ a.e. sample path $\omega$ the exit point $\omega_{+}$is uniquely ergodic. Also, from now on, let $L=L_{T}$ be the drift of the $\mu$ with respect to the Teichmüller metric $d_{T}$ (see Theorem 6.5).

Theorem 3 can be reformulated as follows. For a pseudo-Anosov element $\phi$ let $\gamma_{\phi}$ be a unit speed parametrization of its axis in $\mathcal{T}(S)$ and $l(\phi)$ its translation length in $\mathcal{T}(S)$. Choose moreover a basepoint $o \in \mathcal{T}(S)$. For $\zeta>0$ and a subset $W$ of $\mathcal{T}(S)$ let $N_{\zeta}(W)$ be the $\zeta$-neighborhood of $W$ for the Teichmüller metric.

Theorem 6.8. There is a number $\zeta>0$ with the following property. Let $W \subset \mathcal{T}(S)$ be an $\operatorname{Mod}(S)$ invariant open subset containing an axis of a pseudo-Anosov. Then for each $R>0$, there exists $c=c(W, R)>0$ such that

$$
\begin{gathered}
P\left\{\omega \in \operatorname{Mod}(S)^{\mathbb{N}} \mid \omega_{n} \text { is } p-A\right. \text { and } \\
\left.l\left(\omega_{n}\right)^{-1}\left|\left\{t \in\left[0, l\left(\omega_{n}\right)\right]: \gamma_{\omega_{n}}(t-R, t+R) \subset N_{\zeta} W\right\}\right|>c\right\} \\
\rightarrow 1 \quad(n \rightarrow \infty)
\end{gathered}
$$

To prove Theorem 6.8 we first present two recurrence results for random geodesics, whose proof we defer until the end of the section. These results can be viewed as weak versions of Birkhoff's ergodic theorem for random rays in Teichmüller space.

Proposition 6.9. There is a number $K>0$ with the following property. For every $0<a<b$ and $M>0$, for $P$ almost every sample path $\omega$ there 
is an $n_{0}>0$ such that for all $n>n_{0} \tau_{o, \omega_{+}}([a n, b n])$ contains a connected subsegment of length $M$ contained in $N_{K} \operatorname{Mod}(S)$ o.

Proposition 6.10. Let $W \subset \mathcal{T}(S)$ be an $\operatorname{Mod}(S)$ invariant open subset that contains an axis of a pseudo-Anosov. Then for all $R>0$ there exists a $\hat{c}=\hat{c}(R)>0$ such that for almost every sample path $\omega$ we have:

$$
\liminf \frac{1}{T}\left|\left\{t \in[0, T] \mid \tau_{o, \omega_{+}}[t-R, t+R] \subset W\right\}\right|>\hat{c}
$$

where $\omega_{+}=\operatorname{bnd}_{T}(\omega) \in \mathcal{P} \mathcal{M L}$.

We will use Proposition 6.9 together with Proposition 6.2 to prove our analogue of Proposition 6.2 for $\mathcal{T}(S)$.

Proposition 6.11. There exists a number $\zeta>0$ with the following property. For almost every sample path $\omega$, and each $\varepsilon$, there exists a number $n_{0}>0$ such that for $n \geq n_{0}, \omega_{n}$ is a pseudo-Anosov mapping class with translation length $L n / 2<l\left(\omega_{n}\right)<2 L n$, and the axis of $\omega_{n}$ passes within $\zeta$ of $\tau_{o, \omega_{+}}(t)$ for every $t \in[\varepsilon L n,(1-\varepsilon) L n]$.

Proof. The curve complex $X=\mathcal{C}(S)$ is a separable, Gromov hyperbolic geodesic metric space, and any two points in $X \cup \partial X$ can be connected by a geodesic ray [1]. The mapping class group $G=\operatorname{Mod}(S)$ acts on it as a nonelementary group of isometries. Pseudo-Anosov elements act as loxodromics. Thus by Theorem 6.1, there is an $L^{\prime}>0$ such that

$$
d_{\mathcal{C}(S)}\left(\omega_{n} x, x\right) / n \rightarrow L^{\prime}
$$

for $P$ almost every sample path $\omega$ and every $x \in \mathcal{C}(S)$. Moreover, $P$ almost every sample path $\omega$ converges to some $\omega_{+} \in \partial \mathcal{C}(S)$ (the Gromov boundary of $\mathcal{C}(S)$ ), and there is a unit speed geodesic ray $\alpha$ starting at $x$ and converging to $\omega_{+}$such that $d\left(\alpha\left(L^{\prime} n\right), \omega_{n} x\right) / n \rightarrow 0$. We refer to [33, 21] for a detailed discussion with a comprehensive list of references.

Let $\pi: \mathcal{T}(S) \rightarrow \mathcal{C}(S)$ be the coarsely well-defined map sending $x$ to a shortest curve on $x$. Then for a uniform constant $A>1, \pi$ is $A$ quasiLipschitz (i.e. $d_{\mathcal{C}(S)}(\pi(x), \pi(y)) \leq A d_{\mathcal{T}}(x, y)+A$ for all $x, y \in \mathcal{T}(S)$ ), and it sends Teichmüller geodesics to $A$ quasi-geodesics in $\mathcal{C}(S)$ [24. Moreover, if $g \in \operatorname{Mod}(S)$ is pseudo-Anosov, and if $\gamma$ is the axis of $g$ in $\mathcal{T}(S)$, then $\pi(\gamma)$ is an $A$-quasi-axis for the action of $\operatorname{Mod}(S)$ on $\mathcal{C}(S)$. This means that it is a $g$-quasi-invariant $A$-quasi-geodesic in $\mathcal{C}(S)$.

Furthermore, by Theorem 4.2 of [27], Teichmüller geodesics in the thick part of Teichmüller space are uniformly contracting, and this contraction can be traced in the curve graph [10]. This means that for each $K>0$ there is a number $D=D(K)>0$, and for every $\kappa>0$ there is a number $\kappa^{\prime}>0$ with the following property. Let $\alpha_{1}, \alpha_{2}$ be Teichmüller geodesics with $\alpha_{1}(t-D, t+D) \subset N_{K} \operatorname{Mod}(S) o$ and $d_{\mathcal{C}(S)}\left(\pi\left(\alpha_{1}(t)\right), \pi\left(\alpha_{2}(t)\right)\right)<\kappa$; then $\left.d_{T}\left(\alpha_{1}(t), \alpha_{2}(t)\right)\right)<\kappa^{\prime}$.

Let as before $L$ be the drift of $\mu$ with respect to the Teichmüller metric $d_{T}$. For $P$ almost every sample path $\omega$ there exists a unit speed geodesic 
ray $\tau=\tau_{o, \omega_{+}}$in $\mathcal{T}(S)$ based at $o$ and a unit speed geodesic ray $\alpha$ in $\mathcal{C}(S)$ based at $o^{\prime}=\pi(o)$ such that

$$
d_{T}\left(\tau(L n), \omega_{n} o\right) / n \rightarrow 0 \text { and } d_{C(S)}\left(\alpha\left(L^{\prime} n\right), \omega_{n} o^{\prime}\right) / n \rightarrow 0 .
$$

Consequently, since $\pi: \mathcal{T}(S) \rightarrow \mathcal{C}(\mathcal{S})$ is coarsely Lipschitz we have

$$
d_{\mathcal{C}(S)}\left(\pi(\tau(L n)), \alpha\left(L^{\prime} n\right)\right) / n \rightarrow 0 .
$$

Let $\gamma_{n}$ be the axis of $\omega_{n}$ if $\omega_{n}$ is pseudo-Anosov and $o$ otherwise. Then $\pi\left(\gamma_{n}\right)$ is an $A$ quasi-axis for the action of $\omega_{n}$ on $\mathcal{C}(S)$, and $\pi \circ \tau$ is an $A$ quasi-geodesic. By Proposition 6.2, there is a number $\kappa>0$ such that for $P$ a.e. sample path $\omega$ and every $\varepsilon>0$ there is an $n_{0}>0$ such that for each $n \geq n_{0}$, every point $p \in \pi \circ \tau$ with

$$
\varepsilon L^{\prime} n \leq d_{\mathcal{C}(S)}\left(p, o^{\prime}\right) \leq(1-\varepsilon) L^{\prime} n
$$

is within $\kappa$ of some point of $\pi\left(\gamma_{n}\right)$. Now, if $t \in[3 \varepsilon L n,(1-3 \varepsilon) L n]$ is any integer and $n$ is large enough, using the estimate (13) we have:

$$
\begin{gathered}
d_{\mathcal{C}(S)}\left(o^{\prime}, \pi(\tau(t))\right) \leq d_{\mathcal{C}(S)}\left(o^{\prime}, \alpha\left(\frac{L^{\prime}}{L} t\right)\right)+d_{\mathcal{C}(S)}\left(\alpha\left(\frac{L^{\prime}}{L} t\right), \pi(\tau(t))\right) \\
\leq \frac{L^{\prime}}{L} t+\varepsilon L^{\prime} n \leq(1-3 \varepsilon) L^{\prime} n+\varepsilon L^{\prime} n \leq(1-\varepsilon) L^{\prime} n
\end{gathered}
$$

and similarly

$$
d_{\mathcal{C}(S)}\left(o^{\prime}, \pi(\tau(t))\right) \geq \varepsilon L^{\prime} n
$$

Thus, $\pi(\tau(t))$ is within $\kappa$ of some point of $\pi\left(\gamma_{n}\right)$, and if

$$
\tau([t-D, t+D]) \in N_{K} \operatorname{Mod}(S) o
$$

we have that $\tau(t)$ is within $\kappa^{\prime}$ of some point of $\gamma_{n}$. Now note that by Proposition 6.9, for $P$ almost every $\omega$ and for all large enough $n$ there are

$$
t_{1} \in[3 \varepsilon L n, 4 \varepsilon L n]
$$

and

with

$$
t_{2} \in[(1-4 \varepsilon) L n,(1-3 \varepsilon) L n]
$$

$$
\tau\left(\left[t_{i}-D, t_{i}+D\right]\right) \in N_{K} \operatorname{Mod}(S) o
$$

Thus $\tau\left(t_{i}\right)$ are within $\kappa^{\prime}$ of some point of $\gamma_{n}$ and so by [30], $\tau\left(\left[t_{1}, t_{2}\right]\right)$ is within $\kappa^{\prime \prime}$ of $\gamma_{n}$ where $\kappa^{\prime \prime}$ depends only on $\kappa^{\prime}$.

Finally, we will use Propositions 6.11 and 6.10 to prove Theorem 6.8 and hence Theorem 3 .

Proof of Theorem 6.8. Let $\zeta>0$ be the constant guaranteed by Proposition 6.11. Denote as before by $L$ the drift of the random walk acting on $\mathcal{T}(S)$.

Let $W \subset \mathcal{T}(S)$ be an open $\operatorname{Mod}(S)$-invariant set which contains the axis of a pseudo-Anosov element. Let $R>0$ and let $0<\hat{c}(R+100 \zeta)<1 / 10$ be the constant guaranteed by Proposition 6.10 for $W$ and $R+100 \zeta$ in place of $R$. Let $\varepsilon=\hat{c} / 20<1 / 200$. 
For each $N>0$ let $\Omega_{1}(N)$ be the set of all sample paths $\omega$ such that

$$
\frac{1}{T}\left|\left\{t \in[0, T]: \tau_{o, \omega_{+}}[t-R-100 \zeta, t+R+100 \zeta] \subset W\right\}\right|>\hat{c}
$$

for all $T>(1-\varepsilon) L N$.

Let $\Omega_{2}(N)$ consist of all sample paths $\omega$ such that for all $n>N, \omega_{n}$ is a pseudo-Anosov with the following properties. The translation length of $\omega_{n}$ is contained in $[L n / 2,2 L n]$, and its axis passes within $\zeta$ of $\tau_{o, \omega_{+}}(t)$ for every $t \in[\varepsilon L n,(1-\varepsilon) L n]$. Define $\Omega(N)=\Omega_{1}(N) \cap \Omega_{2}(N)$.

Let $\omega \in \Omega(N)$ and let $n \geq N$. Since $\omega \in \Omega_{2}(N)$, if $t \in[\varepsilon L n,(1-\varepsilon) L n]$ then the axis of $\omega_{n}$ passes within $\zeta$ of $\tau_{o, \omega_{+}}(t)$. By the definition of $\Omega_{1}(N)$, $[\varepsilon L n,(1-\varepsilon) L n]$ has a (not necessarily connected) subset $I_{n}$ of Lebesgue measure at least

$$
\hat{c}(1-\varepsilon) L n-\varepsilon L n-(2 R+200 \zeta)>\hat{c} L n / 2
$$

which is a union of intervals $J$, each of length at least $2 R+200 \zeta$, such that

$$
\tau_{o, \omega_{+}}(J) \subset W
$$

for each $J$.

Let $I_{n}^{\prime} \subset I_{n}$ be a maximal subset which consists of intervals each of length at least $2 R+100 \zeta$ and any two of which are at least $10 \zeta$ apart. Note, $I_{n}^{\prime}$ has measure at least $\hat{c} L n / 4$. For each interval $J$ of $I_{n}^{\prime}$, the axis of $\omega_{n}$ contains a connected subset of length at least $2 R+98 \zeta$ contained within $\zeta$ of $\tau_{o, \omega_{+}}(J) \subset W$. Moreover, since any two intervals of $I_{n}^{\prime}$ are at least $10 \zeta$ apart, the corresponding subsets of the axis of $\omega_{n}$ contained in their $\zeta$ neighborhood are disjoint. Thus, the axis of $\omega_{n}$ contains a subset of measure at least $\hat{c} L n / 4$ which is a union of intervals of length at least $2 R+98 \zeta$ contained in $N_{\zeta}(W)$. Consequently, the $R$-interior of this subset, i.e. the set of all points which are contained in one of these intervals and whose distance to the boundary is at least $R$, has measure at least $98 \zeta \hat{c} L n / 4(2 R+98 \zeta)$.

Since $\omega_{n}$ has translation length at most $2 L n$, the proportion of the points $t$ on the axis which are midpoints of segments of length $2 R$ entirely contained in $N_{\zeta}(W)$ is at least $\tilde{c}=98 \zeta \hat{c} /(2 R+98 \zeta)$ :

$$
l\left(\omega_{n}\right)^{-1}\left|\left\{t \in[0, l(\phi)]: \gamma_{\omega_{n}}(t-R, t+R) \subset N_{\zeta}(W)\right\}\right|>\tilde{c} .
$$

This holds for each $\omega \in \Omega(N)$ and $n \geq N$. By Proposition 6.11 and Proposition 6.10 we know that $P(\Omega(N)) \rightarrow 1$ as $N \rightarrow \infty$, completing the proof.

It remains to prove Proposition 6.10 and Proposition 6.9. Since by Theorem 6.3, $\omega_{+}$is uniquely ergodic for $P$ almost every $\omega$, and by work of Masur 23. any two Teichmüller geodesics with the same uniquely ergodic vertical foliation are asymptotic, the propositions follow from certain bilateral analogues. Namely, for $p \in \mathcal{T}(S)$ and $\zeta_{1}, \zeta_{2} \in \mathcal{P} \mathcal{M L}$ defining a Teichmüller geodesic with the $\zeta_{i}$ as vertical and horizontal measured geodesic laminations, let $\gamma_{\zeta_{1}, \zeta_{2}, p}$ be a unit speed parametrization of this geodesic such that 
$\gamma_{\zeta_{1}, \zeta_{2}, p}(0)$ is at minimal distance from $p$. We can make this choice in a $\operatorname{Mod}(S)$ equivariant way, i.e. so that $g \gamma_{\zeta_{1}, \zeta_{2}, p}(t)=\gamma_{g \zeta_{1}, g \zeta_{2}, g p}(t)$.

For a bilateral sample path $\omega$ converging to distinct uniquely ergodic $\omega^{ \pm} \in \mathcal{P} \mathcal{M L}$ and $p \in \mathcal{T}(S)$ write $\gamma_{\omega, p}$ instead of $\gamma_{\omega_{-}, \omega_{+}, p}$. We also write $\gamma_{\omega}$ for the trace of the axis in $\mathcal{T}(S)$. Proposition 6.10 follows from the following.

Proposition 6.12. Let $W \subset \mathcal{T}(S)$ be an $\operatorname{Mod}(S)$ invariant open subset that contains an axis of a pseudo-Anosov.

For every $R>0$ there exists a $\tilde{c}=\tilde{c}(R)>0$ such that for $\bar{P}$ almost every bilateral sample path $\omega \in \operatorname{Mod}(S)^{\mathbb{Z}}$ we have:

$$
\lim \inf _{T \rightarrow \infty} \frac{1}{T}\left|\left\{t \in[-T, T]: \gamma_{\omega, o}([t-R, t+R]) \subset W\right\}\right|>\tilde{c}
$$

Proof. For each $K, R>0$ let $\Omega(K, R)$ be the set of sample paths $\omega$ such that there is a Teichmüller geodesic with vertical and horizontal laminations $\omega_{+}$ and $\omega_{-}$, and moreover $\gamma_{\omega, o}([-2 R, 2 R]) \subset W$ and $d\left(o, \gamma_{\omega}\right)<K$.

Lemma 6.13. There is a $K=K(W)>0$ such that $\bar{P}(\Omega(K, R))>0$ for all $R>0$.

Proof. Let $K(W)$ be large enough so that there exists a pseudo-Anosov element $\phi$ with attractive and repellant (projective) measured foliations $\phi^{+}$ and $\phi^{-}$with axis $\gamma_{\phi_{-}, \phi_{+}}$passing within $K / 2$ of $o$ and contained in

$$
W \cap N_{K / 2} \operatorname{Mod}(S) o .
$$

Let $\gamma_{\phi_{-}, \phi_{+}, o}$ be the associated parametrization for its axis. Let $a>0$ be such that the $a$ neighborhood of the axis of $\phi$ is contained in $W \cap N_{K / 2} \operatorname{Mod}(S) o$.

Filling pairs of laminations are an open subset of $\mathcal{P} \mathcal{M L} \times \mathcal{P} \mathcal{M L}$, and any such pair determines a Teichmüller geodesic with corresponing vertical and horizontal measured foliations. Moreover, if a sequence of such pairs converges to the pseudo-Anosov pair $\left(\phi_{-}, \phi_{+}\right)$, then the corresponding geodesics converge locally uniformly to $\gamma_{\phi_{-}, \phi_{+}, o}$. Thus for every $R>0$ there are open neighborhoods $U^{ \pm} \subset \mathcal{P} \mathcal{M L}$ of $\phi^{ \pm}$such that for all $\zeta_{1} \in U^{+}$and $\zeta_{2} \in U^{-}$ we have

$$
d\left(\gamma_{\zeta_{1}, \zeta_{2}, o}(t), \gamma_{\phi_{-}, \phi_{+}, o}(t)\right)<a
$$

for all $t \in[-2 R, 2 R]$.

Let $\Omega^{\prime}(K, R)$ be the set of all sample paths $\omega$ with $\omega^{ \pm} \in U^{ \pm}$. By definition, $\Omega^{\prime}(K, R) \subset \Omega(K, R)$. Since the $U^{ \pm}$are open subsets of $\mathcal{P} \mathcal{M L}$ and the harmonic measure $\nu$ has full support on $\mathcal{P} \mathcal{M L}$, we have $\nu\left(U^{ \pm}\right)>0$ and hence $\bar{P}\left(\Omega^{\prime}(K, R)\right)>0$ and thus $\bar{P}(\Omega(K, R))>0$.

Let $\sigma: G^{\mathbb{Z}} \rightarrow G^{\mathbb{Z}}$ be the left Bernoulli shift: $\sigma(\omega)_{n}=\omega_{n+1}$. By basic symbolic dynamics, $\sigma$ is invertible, measure preserving and ergodic with respect to $\mu^{\mathbb{Z}}$. Therefore,

$$
U=T \circ \sigma \circ T^{-1}
$$


is invertible, measure preserving and ergodic with respect to $\bar{P}$. Note that for each $n \in \mathbb{Z}$,

and more generally

$$
(U \omega)_{n}=\omega_{1}^{-1} \omega_{n+1}
$$

$$
\left(U^{k} \omega\right)_{n}=\omega_{k}^{-1} \omega_{n+k}
$$

Let $K>0$ be as in Lemma 6.13. Since $W$ and $d_{T}$ are $\operatorname{Mod}(S)$ invariant, we have that $U^{k} \omega \in \Omega(K, R)$ if and only if $\gamma_{\omega, \omega_{k} o}([-2 R, 2 R]) \subset W$ and $d\left(\omega_{k} o, \gamma_{\omega}\right)<K$.

Without loss of generality, we can assume that $R>100 K$. Let $s_{i}(\omega)=$ $d_{T}\left(\omega_{i} o, \gamma_{\omega, o}(0)\right)$. By Theorem 6.5, for almost all $\bar{P}$ almost all $\omega$ we have $s_{i}(\omega) / i \rightarrow L$.

If $U^{i} \omega \in \Omega(K, R)$ then there is some $t_{i}(\omega)$ with $\left|t_{i}(\omega)-s_{i}(\omega)\right|<2 K$ and

$$
\gamma_{o, \omega}\left[t_{i}(\omega)-2 R, t_{i}(\omega)+2 R\right] \subset W .
$$

Define

$$
I(\omega)=\cup_{\left\{i \mid U^{i}(\omega) \in \Omega(K, R)\right\}}\left[t_{i}(\omega)-R, t_{i}(\omega)+R\right] .
$$

We need to show that $I(\omega)$ has positive density in $\mathbb{R}$, ie that

$$
\lim \inf _{\rho \rightarrow \infty}|I(\omega) \cap[-\rho, \rho]| / 2 \rho>0 .
$$

Since

$$
\left|t_{i}(\omega)-s_{i}(\omega)\right|<2 K
$$

whenever $U^{i} \omega \in \Omega(K, R)$, it suffices to show that

$$
I^{\prime}(\omega)=\cup_{\left\{i \mid U^{i}(\omega) \in \Omega(K, R)\right\}}\left[s_{i}(\omega)-R, s_{i}(\omega)+R\right]
$$

has positive density in $\mathbb{R}$.

Let

$$
d=\sup \left\{d_{T}(o, g o) \mid g \in \operatorname{supp}(\mu)\right\}
$$

(this is finite since $\mu$ has finite support). Then by $\operatorname{Mod}(S)$ invariance of $d_{T}$, for all $\omega$ and $k$ we have $d_{T}\left(\omega_{k+1} o, \omega_{k}\right)<d$. Thus for each

$$
t>d_{T}\left(o, \gamma_{\omega}\right)
$$

there is a $n_{t}(\omega) \in \mathbb{N}$ with

$$
\left|s_{n_{t}(\omega)}(\omega)-t\right|<d .
$$

If $q>0$ and if $n^{\prime}$ is a number with $\left|n_{t}(\omega)-n^{\prime}\right| \leq q$ then

$$
d_{T}\left(\omega_{n_{t}(\omega)} o, \omega_{n^{\prime}} O\right) \leq q d
$$

and hence $\operatorname{dist}\left(t, I_{n^{\prime}}^{\prime}(\omega)\right) \leq q d+d$. Thus if $n^{\prime}$ is such that $U^{n^{\prime}}(\omega) \in \Omega(K, R)$ then $t \in N_{q d+d}\left(I^{\prime}(\omega)\right)$.

Now, for $A>0$ assume that $t$ is such that $d\left(t, I^{\prime}(\omega)\right)>A d+d$. Then there exists an integer $n>0$ with $U^{i} \omega \notin \Omega(K, R)$ for any $i \in[n-A, n+A]$ and $\left|t-d\left(\omega_{n} o, \gamma_{\omega}(0)\right)\right|<d$.

Let $\Omega_{0} \subset \operatorname{Mod}(S)^{\mathbb{Z}}$ be the $\bar{P}$ full measure set consisting of $\omega$ such that $d\left(\omega_{m} o, o\right) / m \rightarrow L$ as $m \rightarrow \pm \infty$. For $\omega \in \Omega_{0}$ and large enough $t$ (depending on $\omega)$ we have $n_{t}(\omega) \in[9 t /(10 L), 11 t /(10 L)]$. 
Define

$$
\Lambda(A, K, R)=\left\{\omega \mid U^{i} \omega \notin \Omega(K, R) \text { for any }|i| \leq A\right\} .
$$

Let $\Upsilon_{A, K, R}(\omega)$ be the set of $k \in \mathbb{Z}$ with $U^{i} \omega \notin \Omega(K, R)$ for any

$$
k-A \leq i \leq k+A .
$$

By definition, $U^{k} \omega \in \Lambda(A, K, R)$ if and only if $k \in \Upsilon_{A, K, R}(\omega)$. Thus any large enough $t$ with $d\left(t, I^{\prime}(\omega)\right)>A d+d$ is contained in

$$
\left[d_{T}\left(\omega_{k} o, \gamma_{\omega}(0)\right)-d, d_{T}\left(\omega_{k} o, \gamma_{\omega}(0)\right)+d\right]
$$

for some $k<11 t /(10 L)$ with $k \in \Upsilon_{A, K, R}(\omega)$. By the Birkhoff ergodic theorem, $\bar{P}(\Lambda(A, K, R)) \rightarrow 0$ as $A \rightarrow \infty$.

Also by the Birkhoff ergodic theorem, for almost every $\omega$,

$$
\lim _{N \rightarrow \infty}\left|[-N, N] \cap \Upsilon_{A, K, R}(\omega)\right| /(2 N)=\bar{P}(\Lambda(A, K, R)) .
$$

Thus for large enough $T$ (depending on $A$ ) the Lebesgue measure of the set

$$
\left\{t \in[-T, T] \mid d\left(t, I^{\prime}(\omega)\right)>A d+d\right\}
$$

is less than $3 d P(\Lambda(A, K, R))(11 T / 10 L)$.

Therefore the density of $t \in \mathbb{R}$ with $d\left(t, I^{\prime}(\omega)\right)>A d+d$ is less than $3 d P(\Lambda(A, K, R)) / L$ which is less than $1 / 10$ for large enough $A$. Thus for large enough $A$ the $A d+d$ neighborhood of $I^{\prime}(\omega)$ has density at least $9 / 10$ in $\mathbb{R}$ so $I^{\prime}(\omega)$ itself has density at least $c=\frac{9}{10(A d+d)}>0$ in $\mathbb{R}$. This completes the proof of Proposition 6.12 .

Proposition 6.9 follows from the following bilateral statement.

Proposition 6.14. There is a $K>0$ with the following property. For every $a<b$ and $M>0$, for $P$ almost every sample path $\omega$ there is an $n_{0}>0$ such that for all $n>n_{0} \gamma_{\omega, o}(c n, d n)$ contains a connected subsegment of length $M$ contained in $N_{K} \operatorname{Mod}(S) o$.

Proof. Let $\Omega(M, K, R)$ be the set of sample paths $\omega \in \operatorname{Mod}(S)^{\mathbb{Z}}$ such that $d_{T}\left(o, \gamma_{\omega}\right)<R / 10$ and $\gamma_{\omega, o}(t-M, t+M) \subset N_{K} \operatorname{Mod}(S) o$ for some $t \in$ $(-R / 2+M, R / 2-M)$.

Lemma 6.15. There is a $K>0$ such that for all $M>0$ there is an function $f$ with $\lim _{R \rightarrow \infty} f(R)=0$ and $\bar{P}(\Omega(M, K, R))>1-f(R)$.

We first continue with the proof of Proposition 6.14 assuming Lemma 6.15 and will prove Lemma 6.15 afterwards.

Assume without loss of generality that $a>0$ (notation as in the statement of Proposition 6.14). Let as before $\Omega_{0} \subset \operatorname{Mod}(S)^{\mathbb{Z}}$ denote the $\bar{P}$ full measure set of all $\omega$ such that

$$
d_{T}\left(\omega_{i} o, o\right) / i \rightarrow L
$$

and consider $\omega \in \Omega_{0}$. Choose $R>0$ large enough so that

$$
1-f(R)>(b-a) /(10 a+10 b)
$$


Note, $U^{k} \omega \in \Omega(M, K, R)$ if and only if $d_{T}\left(\omega_{i} o, \gamma_{\omega}\right)<R / 10$ and

$$
\gamma_{\omega, \omega_{i} o}(t-M, t+M) \subset N_{K} \operatorname{Mod}(S) o
$$

for some $t \in(-R / 2+M, R / 2-M)$. This implies that

$$
\gamma_{\omega, o}\left(t_{i}(\omega)-M, t_{i}(\omega)+M\right) \subset N_{K} \operatorname{Mod}(S) o
$$

for some $t_{i}(\omega)$ with $\left|t_{i}(\omega)-d\left(\omega_{i} o, \gamma_{\omega, o}(0)\right)\right|<R / 10$.

Let $s_{i}(\omega)=d_{T}\left(\omega_{i} o, \gamma_{\omega, o}(0)\right)$ and let again

$$
d=\sup \left\{d_{T}(o, g o) \mid g \in \operatorname{supp}(\mu)\right\} .
$$

As in the proof of Proposition 6.12, note for every $t>d_{T}\left(o, \gamma_{\omega}\right)$ there is some $i(t)$ with $\left|t-s_{i(t)}(\omega)\right|<d$. Hence, for large enough (depending on $\omega$ ) $n$ if there is an $i$ with

$$
U^{i} \omega \in \Omega(M, K, R)
$$

and

$$
(2 a+b) n / 3 \leq s_{i}(\omega) \leq(a+2 b) n / 3
$$

then

$$
\gamma_{\omega, o}([a n, b n]) \cap N_{K} \operatorname{Mod}(S) o
$$

has a connected segment of length $M$.

Moreover, $s_{i}(\omega) / i \rightarrow L$. Thus, for large enough $n$, we have

$$
(2 a+b) n / 3 \leq s_{i}(\omega) \leq(a+2 b) n / 3
$$

for every $i$ with

$$
\frac{(3 a+2 b) n}{5 L} \leq i \leq \frac{(2 a+3 b) n}{5 L}
$$

Hence, if $\gamma_{\omega, o}([a n, b n]) \cap N_{K} \operatorname{Mod}(S) o$ does not have a length $M$ connected segment we have

$$
U^{i} \omega \notin \Omega(M, K, R)
$$

for any

$$
\frac{(3 a+2 b) n}{5 L} \leq i \leq \frac{(2 a+3 b) n}{5 L}
$$

If this holds for infinitely many $n$ we have

$$
\lim \inf _{N \rightarrow \infty} \frac{\left|\left\{i \in[0, N-1] \mid U^{i} \omega \in \Omega(M, K, R)\right\}\right|}{N} \leq 1-\frac{b-a}{2 a+3 b}
$$

On the other hand, by the Birkhoff ergodic theorem we have:

$$
\begin{aligned}
& \lim _{N \rightarrow \infty} \frac{\left|\left\{i \in[0, N-1] \mid U^{i} \omega \in \Omega(M, K, R)\right\}\right|}{N} \\
= & \bar{P}(\Omega(M, K, R))>1-(b-a) /(10 a+10 b)
\end{aligned}
$$

giving a contradiction.

Finally, we prove Lemma 6.15. 
Proof of Lemma 6.15. Clearly the $\bar{P}$ measure of $\omega \in \operatorname{Mod}(S)^{\mathbb{Z}}$ such that $d_{T}\left(o,\left[\omega_{-}, \omega_{+}\right]\right)<R / 10$ converges to 1 with $R$. Thus it suffices to show that for each $M>0$ the $\bar{P}$ measure of $\omega$ such that $\gamma_{\omega, o}([-R, R]) \cap N_{K} \operatorname{Mod}(S) o$ contains a length $M$ connected subsegment converges to 1 with $R$. Let $\Lambda(M, K)$ be the set of $\omega$ such that $d_{T}\left(o, \gamma_{\omega}\right)<K$ and

$$
\gamma_{\omega, o}([-M, M]) \subset N_{K} \operatorname{Mod}(S) o .
$$

The following is similar to Lemma 6.13 .

Claim 6.16. There is a $K>0$ such that $\bar{P}(\Lambda(M, K))>0$ for all $M$.

Proof. Let $K>0$ be large enough so that there exists a pseudo-Anosov element $\phi$ with attractive and repellant (projective) measured foliations $\phi^{+}$and $\phi^{-}$with axis $\gamma_{\phi_{-}, \phi_{+}}$passing within $K / 2$ of $o$ and contained in $N_{K / 2} \operatorname{Mod}(S) o$. Let $\gamma_{\phi_{-}, \phi_{+}, o}$ be the associated parametrization for its axis. Then there are open neighborhoods $U^{ \pm} \subset \mathcal{P} \mathcal{M L}$ of $\phi^{ \pm}$such that for all $\zeta_{1} \in U^{+}$and $\zeta_{2} \in U^{-}, d_{T}\left(\gamma_{\zeta_{1}, \zeta_{2}, o}(t), \gamma_{\phi_{-}, \phi_{+}, o}(t)\right)<K / 2$ for all $t \in[-2 M, 2 M]$.

Let $\Lambda^{\prime}(M, K)$ be the set of all sample paths $\omega$ with $\omega^{ \pm} \in U^{ \pm}$. By definition, $\Lambda^{\prime}(M, K) \subset \Lambda(M, K)$. Since the $U^{ \pm}$are open subsets of $\mathcal{P} \mathcal{M L}$ and the harmonic measure $\nu$ has full support on $\mathcal{P} \mathcal{M L}$ we have $\nu\left(U^{ \pm}\right)>0$ and hence $\bar{P}\left(\Lambda^{\prime}(M, K)\right)>0$ and thus $\bar{P}(\Lambda(M, K))>0$.

Note, $U^{i} \omega \in \Lambda(M, K)$ if and only if

$$
d_{T}\left(o, \gamma_{\omega}\right)<K
$$

and

$$
\gamma_{\omega, \omega_{i} o}([-M, M]) \subset N_{K} \operatorname{Mod}(S) o .
$$

Note, $d_{T}\left(o, \omega_{i} o\right) \leq d i$ and hence if

$$
U^{i} \omega \in \Lambda(M, K)
$$

for some $i$ with

$$
0 \leq i \leq \frac{R-M-2 K}{2 d}
$$

then

$$
\gamma_{\omega, o}([-R, R]) \cap N_{K} \operatorname{Mod}(S) o
$$

contains a length $M$ connected subsegment. By the Birkhoff ergodic theorem, the $\bar{P}$ measure of sample paths $\omega$ such that $U^{i} \omega \notin \Lambda(M, K)$ for all $i$ with

$$
0 \leq i \leq \frac{R-M-2 K}{2 d}
$$

converges to 0 with $R$ completing the proof. 


\section{REFERENCES}

[1] B. Bowditch, Tight geodesics in the curve complex. Invent. Math. 171 (2008), no. 2, 281-300.

[2] J. Brock, Weil-Petersson translation distance and volumes of mapping tori, Comm. Anal. Geom. 11 (2003), pp. 987-999.

[3] J. Brock, K. Bromberg, Inflexibility, Weil-Peterson distance, and volumes of fibered 3-manifolds. arXiv:1412.0733, to appear in Math. Res. Letters.

[4] J. Brock, R. Canary, Y. Minsky, The classification of Kleinian surface groups, II: The Ending Lamination Conjecture. Ann. Math. 176 (1) (2012), pp. 1-149.

[5] P. Buser, A note on the isoperimetric constant. Ann. Scient. Ec. Norm. Sup. 15 (1982), 213-230.

[6] P. Buser, Geometry and spectra of compact Riemann surfaces. Birkhäuser, Boston 1992.

[7] F. Dahmani, C. Horbez, Spectral theorems for random walks on mapping class groups and $\operatorname{Out}\left(F_{N}\right)$. arXiv:1506.06790.

[8] J. Dodziuk, B. Randol, Lower bounds for $\lambda_{1}$ on a finite-volume hyperbolic manifold. J. Diff. Geom. 24 (1986), 133-139.

[9] V. Gadre, J. Maher, The stratum of random mapping classes. arXiv:1607.01281.

[10] U. Hamenstädt, Stability of quasi-geodesics in Teichmüller space. Geom. Dedicata 146 (2010), 101-116.

[11] U. Hamenstädt, Bowen's construction for the Teichmüller flow. J. Mod. Dynamics 7 (2013), 489-526.

[12] U. Hamenstädt, Counting periodic orbits in the thin part of strata. Preprint 2016.

[13] U. Hamenstädt, Small eigenvalues and thick-thin decomposition in negative curvature. Preprint 2018.

[14] V. A. Kaimanovich, The Poisson formula for groups with hyperbolic properties. Ann. of Math. (2) 152 (2000), 659-692.

[15] V.A. Kaimanovich, H. Masur, The Poisson boundary of the mapping class group. Invent. Math. 125 (1996), 221-264.

[16] S. Kojima, G. McShane, Normalized entropy versus volume for pseudo-Anosovs. arXiv:1411.6350

[17] M. Lackenby, Heegaard splittings, the virtually Haken conjecture and Property $(\tau)$. Invent. Math. 164 (2006), 317-369.

[18] A. Lenzhen, J. Souto, in preparation.

[19] L. Louder, J. Souto, Diameter and spectral gap for planar graphs. arXiv:1204.4435.

[20] J. Maher, Random walks on the mapping class group. Duke Math. J. 156 (2011), 429-486.

[21] J. Maher, G. Tiozzo, Random walks on weakly hyperbolic groups. arXiv:1410.4173.

[22] T. Mantuano, Discretization of Compact Riemannian Manifolds Applied to the Spectrum of Laplacian, Ann. Global Anal. \& Geom. 27 (2005), 33-46.

[23] H. Masur, Uniquely ergodic quadratic differentials. Comment. Math. Helv. 55 (1980), $255-266$

[24] H. Masur, Y. Minsky, Geometry of the complex of curves I: Hyperbolicity. Invent. Math. 138 (1) (1999), 103-149.

[25] H. Masur, Y. Minsky, Geometry of the complex of curves II: Hierarchical structure. Geom. Funct. Anal. 10 (4) (2000), 902-974.

[26] Y. Minsky, Teichmüller geodesics and end invariants of hyperbolic 3-manifold. Topology 32 (1993), 625-647.

[27] Y. Minsky, Quasi-projections in Teichmüller space. J. Reine Angew. Math. 473 (1996), $121-136$.

[28] Y. Minsky, The classification of Kleinian surface groups, I: Models and bounds, Ann. Math. 171 (1) (2010), pp. 1-107. 
[29] R. Penner, J. Harer, Combinatorics of train tracks. Ann. Math. Studies, Princeton Univ. Press, Princeton 1992.

[30] K. Rafi, Hyperbolicity in Teichmüller space. Geom. Topol. 18 (2014), 3025-3053.

[31] I. Rivin, Statistics of Random 3-Manifolds occasionally fibering over the circle. arXiv:1401.5736.

[32] R. Schoen, A lower bound for the first eigenvalue of a negatively curved manifold J. Differential Geom. 17 (2) (1982), pp.233-238.

[33] G. Tiozzo, Sublinear deviation between geodesics and sample paths. Duke Math. J. 164 (2015), 511-539.

[34] N. White, Spectral bounds on closed hyperbolic 3-manifolds, J. London Math. Soc. 87 (2013), 837-852.

Hyungryul Baik, Department of Mathematical Sciences, KAIST 291 Daehakro, Yuseong-gu, 34141 Republic of Korea

Ilya Gekhtman, Department of Mathematics, Yale University, 10 Hillhouse Ave, New Haven, Connecticut 06520, USA

Ursula Hamenstädt, Universität Bonn, Endenicher Allee 60, 53115 Bonn, Germany

e-mail:

Hyungryul Baik: hrbaik@kaist.ac.kr

Ilya Gekhtman: ilya.gekhtman@yale.edu

Ursula Hamenstädt: ursula@math.uni-bonn.de 